ARTICLE

https://doi.org/10.1038/s41467-019-11875-6

\title{
Exploring use of unsupervised clustering to associate signaling profiles of GPCR ligands to clinical response
}

\author{
Besma Benredjem 1,2,11, Jonathan Gallion 3,11, Dennis Pelletier ${ }^{4}$, Paul Dallaire ${ }^{1,2}$, Johanie Charbonneau², \\ Darren Cawkill ${ }^{4}$, Karim Nagi (10 5, Mark Gosink4, Viktoryia Lukasheva6 ${ }^{6}$, Stephen Jenkinson ${ }^{4,8}$, Yong Ren ${ }^{4,9}$, \\ Christopher Somps ${ }^{4}$, Brigitte Murat ${ }^{6}$, Emma Van Der Westhuizen (10) 6,10, Christian Le Gouill ${ }^{6}$, Olivier Lichtarge ${ }^{3}$, \\ Anne Schmidt ${ }^{4}$, Michel Bouvier (iD ${ }^{6} \&$ Graciela Pineyro ${ }^{1,2}$
}

Signaling diversity of $\mathrm{G}$ protein-coupled (GPCR) ligands provides novel opportunities to develop more effective, better-tolerated therapeutics. Taking advantage of these opportunities requires identifying which effectors should be specifically activated or avoided so as to promote desired clinical responses and avoid side effects. However, identifying signaling profiles that support desired clinical outcomes remains challenging. This study describes signaling diversity of mu opioid receptor (MOR) ligands in terms of logistic and operational parameters for ten different in vitro readouts. It then uses unsupervised clustering of curve parameters to: classify MOR ligands according to similarities in type and magnitude of response, associate resulting ligand categories with frequency of undesired events reported to the pharmacovigilance program of the Food and Drug Administration and associate signals to side effects. The ability of the classification method to associate specific in vitro signaling profiles to clinically relevant responses was corroborated using $\beta 2$-adrenergic receptor ligands.

\footnotetext{
${ }^{1}$ Department of Pharmacology and Physiology, Faculty of Medicine, Université de Montréal, Montréal, QC H3T 1J4, Canada. ${ }^{2}$ CHU Sainte-Justine research center, Montréal, QC H3T 1C5, Canada. ${ }^{3}$ Baylor College of Medicine, Houston, TX 77030, USA. ${ }^{4}$ Pfizer Inc, Groton, CT 06340, USA. ${ }^{5}$ College of Medicine, Member of QU Health, Qatar University, Doha, Qatar. ${ }^{6}$ Institute for Research in Immunology and Cancer, Department of Biochemistry and Molecular Medicine, Université de Montréal, Montréal, QC H3T 1J4, Canada. ${ }^{7}$ Present address: Apollo Therapeutics LLP, Stevenage Bioscience Catalyst, Gunnels Wood Road, Stevenage, SG1 2FX, UK. ${ }^{8}$ Present address: Pfizer Inc, La Jolla, CA 92121, USA. ${ }^{9}$ Present address: Decibel Therapeutics, 1325 Boylston Street, Boston, MA 02215, USA. ${ }^{10}$ Present address: Monash Institute of Pharmaceutical Sciences, Parkville, VIC 3052, Australia. ${ }^{11}$ These authors contributed equally: Besma Benredjem, Jonathan Gallion. Correspondence and requests for materials should be addressed to M.B. (email: michel.bouvier@umontreal.ca) or to G.P. (email: graciela.pineyro.filpo@umontreal.ca)
} 
G protein-coupled receptors (GPCRs) modulate practically every aspect of human physiology and are the target of $\sim 30 \%$ of FDA-approved medicines ${ }^{1}$. When activated these receptors undergo conformational changes 2,3 that determine the type and the magnitude of signals triggered within the cell ${ }^{4}$. This signaling configuration supports ligand-specific activation of the different pathways ${ }^{4}$, and provides a theoretical opportunity for directing pharmacological stimulus toward pathways that underlie desired therapeutic responses and away from those responsible for undesired side effects ${ }^{5,6}$. However, in spite of this promise ${ }^{5}$ development of therapeutic biased ligands has yet to translate into more effective and/or better-tolerated medicines $^{7-11}$.

Different challenges have hindered the development of clinically effective biased ligands. Except for limited examples ${ }^{12-14}$, we ignore the signals underlying desired and undesired clinical responses of GPCR ligands. To access this knowledge and apply it to drug discovery, it is necessary to identify signaling preferences and to associate distinct signaling profiles to desired/undesired clinical outcomes ${ }^{15,16}$. The way in which signaling preferences are currently identified in drug discovery efforts involves calculation of "bias factors", an approach that uses consolidated $(\log (\tau / \mathrm{KA}))$ transduction coefficients to measure the extent to which a ligand preferentially activates one pathway over another ${ }^{17-19}$. This type of evaluation compares signals in a pairwise manner, a dichotomous approach that provides a fragmented view of a ligand's signaling preferences across the multiplicity of pathways. Perhaps more troubling for the use of "bias factors" as descriptors of potential clinical responses is the fact that their estimated magnitudes vary with the calculation method used to produce them ${ }^{15}$. Finally, the same "bias factor" may describe drugs with very different efficacies at the pathways of interest ${ }^{20}$ further questioning the value of these measures as predictors of desired/ undesired in vivo responses. In an effort to circumvent at least some of these limitations, we sought an alternative way to identify signaling preferences and classify GPCR ligands.

One of the most studied examples of how biased signaling may support development of more effective and/or bettertolerated therapeutic agents is that of opioid analgesics. Preclinical models have indicated that $\beta$-arrestin2 ( $\beta$ arr2) knockout mitigates constipation and respiratory depression induced by morphine ${ }^{21}$, pointing to the possibility that $\mathrm{mu}$ opioid receptor (MOR) agonists that preferentially activate $G$ protein signaling over $\beta$ arr 2 recruitment could induce less of these side effects ${ }^{12-14}$. Here, we use this prototypical example to establish that clustering MOR ligands according to similarities in pharmacodynamic parameters for multiple responses, captures their signaling differences and preferences. We show that ligands with similar $G$ protein/ $\beta$ arr responses cluster together, and provide evidence that ligands within different categories display distinct frequencies of gastrointestinal and respiratory events reported to the FDA pharmacovigilance program. Moreover, when ligands are clustered according to either $G$ protein or $\beta$ arr responses both signals directly associate to side effects. The practical value of the classification method proposed is further illustrated by the fact that ligand categories defined by similarity of $G$ protein responses at $\beta 2$-adrenergic receptor $(\beta 2 \mathrm{AR})$ correlate with sympatholytic $\mathrm{CV}$ events and bronchoconstriction.

\section{Results}

Clustering ligands according to pharmacodynamic similarities. We sought a method to identify and group together ligands with overall similarities in a multiplicity of signaling pathways while simultaneously discerning those with overall differences in features, such as efficacy, potency, and signaling preferences. To test the ability of the method to accomplish this task independent of idiosyncrasies in experimental data sets, we generated a set of 320 virtual compounds as variations of 16 prototypical profiles characterized by a combination of pharmacodynamic features across six different readouts (see the Methods section). Profiles are shown in Supplementary Fig. 1. Criteria to classify ligands according to pharmacodynamic similarities were empirically established by generating matrices, in which each ligand was represented by individual logistic $\left(E_{\max }, \mathrm{pEC50}\right)$ or operational $(\log (\tau), \mathrm{pKA}, \log (\tau / \mathrm{KA}))$ parameters, as well as their combinations. Matrices were then subject to nonnegative matrix factorization $(\mathrm{NNMF})^{22}$ to identify essential, nonredundant features, and k-means clustering was subsequently used to classify ligands according to these features ${ }^{23}$. Iterations were used to incorporate the error associated with each mean parameter value, ensuring its propagation throughout the clustering procedure (see the Methods and Supplementary Fig. 2). The result of this procedure was a ligand $\times$ ligand similarity matrix that quantifies how frequently any two compounds clustered together over the iterations. Final similarity matrices were submitted to hierarchical clustering to establish row and column ordering according to similarity, and visualized as heatmaps. Figure 1 shows heatmaps for the progressive associations of parameters leading to identification of $\log (\tau), E_{\max }$, and $\log (\tau / \mathrm{KA})$ as a combination faithfully recreating the 16 profiles initially defined. Operational efficacy $(\tau)$ by itself was not sufficient to fully distinguish ligands with different profiles (Fig. 1a, d). Introducing measures of signaling capacity $\left(E_{\max }\right)$ improved the classification (Fig. 1, e), but discrimination was not optimal unless values for transduction coefficient $\log (\tau / \mathrm{KA})$ were also included (Fig. 1c, f). Unlike $\log (\tau)$ and $E_{\max }$ values, transduction coefficients incorporate potency information ${ }^{24,25}$ and thus provide a different dimension on which ligands can be distinguished. In effect, $\log (\tau / \mathrm{KA})$ coefficients were correlated with logistic potency estimates (pEC50) (Supplementary Fig. 3a), so the classification afforded by $\log (\tau)-E_{\max }-\mathrm{pEC50}$ (Supplementary Fig. 3b, c) was quite similar to the one produced with $\log (\tau)-E_{\max }-\log (\tau / \mathrm{KA})$. Profiles recreated by classifying ligands according to $\log (\tau)$ $E_{\max }-\log (\tau / \mathrm{KA})$, are shown in Supplementary Fig. 4, revealing a minimal number of displaced compounds.

We then applied the proposed classification strategy on experimental data. Multidimensional signaling profiles for this analysis were generated using ten different BRET-based biosensors that monitor ßarr recruitment and G protein signaling. Gprotein signaling was monitored through conformational rearrangements within $\mathrm{Ga}_{\mathrm{i} 1-2} /{ }_{\mathrm{oA}} \beta_{1} \gamma_{2}$ heterotrimers ${ }^{26}$, at the interface of $\mathrm{G} \beta_{1} \gamma_{2} / \mathrm{Kir} 3$ channel subunits ${ }^{27}$, or as changes in cAMP levels ${ }^{28,29}$. $\beta$ arr recruitment to the receptor was assessed for $\beta$ arr1, ßarr2, and $\beta$ arr2 in presence of GRK2, GRK5, or GRK6 to account for possible impact of expression differences between the screening system (HEK 293) and target neurons where GRK levels are higher ${ }^{30}$. Net BRET values obtained in cells coexpressing human MORs (hMOR) and different biosensors in presence/absence of the endogenous ligand Met-Enkephalin (Met-ENK) are shown for reference in Supplementary Fig. 5. Concentration response curves (CRCs) for 10 known opioids (Fig. 2), and 15 novel compounds (Supplementary Fig. 6) identified in the context of a screening campaign at Pfizer Inc. ${ }^{31}$, were then generated and analyzed with the logistic equation and the operational ${ }^{32}$ model. Each ligand was phenotypically described by corresponding $\tau, E_{\mathrm{MAX}}$, and $\log (\tau /$ $\mathrm{KA})$ values $( \pm$ SEM) derived from $5 \mathrm{G}$ protein- and 5 ßarr-related responses (Supplementary Data 1). These were analyzed with $\mathrm{NNMF} / \mathrm{k}$-means clustering as above and represented as heat maps for ligands (Fig. 3a) and for parameters (Fig. 3b). 
a

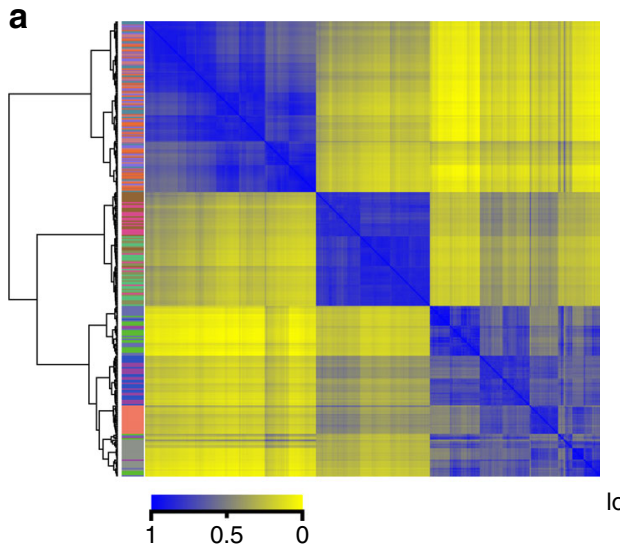

b

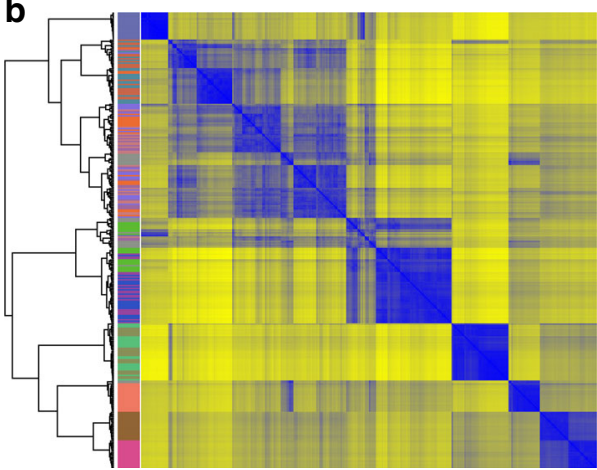

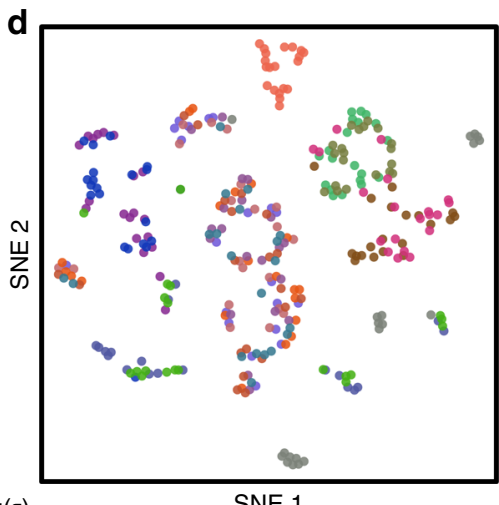

SNE 1

\section{e}

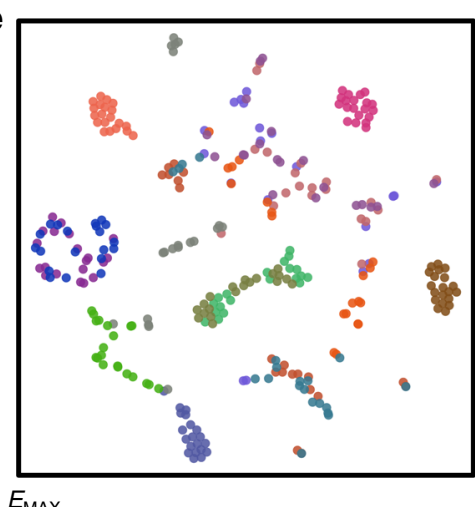

C
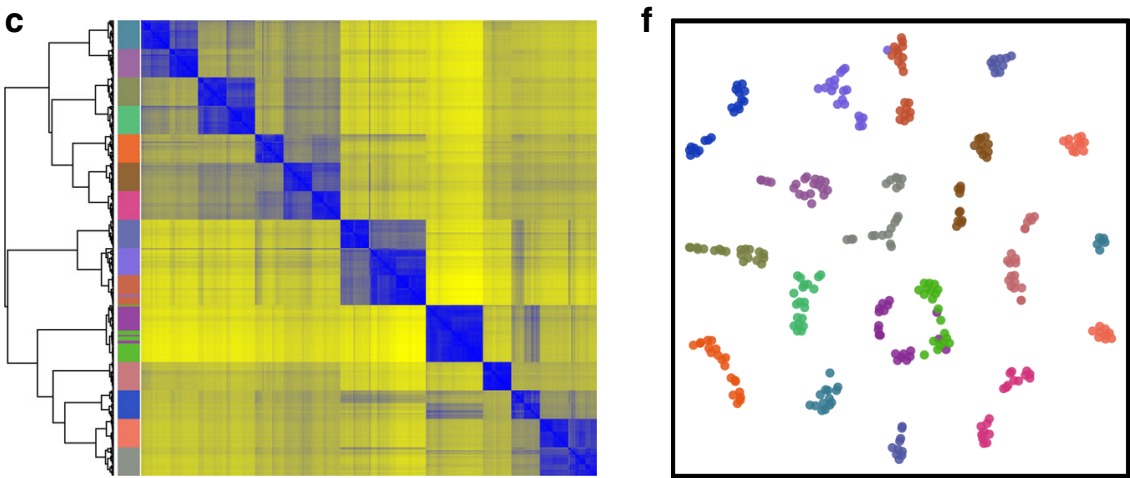

$\log (\tau)$ and $E_{\mathrm{MAX}}$ and $\log \left(\tau / \mathrm{KA}_{\mathrm{A}}\right)$

Fig. 1 Ligands are classified according to similarities in multidimensional signaling profiles using pharmacodynamic parameters. 320 virtual compounds were defined by logistic and operational parameters to represent 16 distinct signaling profiles describing response at six different readouts. Indicated parameters were then subject to NNMF followed by k-means clustering, to produce corresponding similarity matrices that were represented as heatmaps and hierarchical clustering trees (using the R heatmap function with the metric:ward.D2) (a-c) or t-SNE plots (using the R package tsne with default parameters) (d-f). Ligands were color-coded to highlight how different combinations of parameters differentiated compounds originating from the different profiles originally defined

Ligands within the same cluster share quality and magnitude of response. An essential pharmacological question is to identify the pathways and pharmacodynamic properties primarily responsible for ligand clustering. The overall resemblance among relative magnitudes of operational and logistic parameters from different functional readouts is shown in Fig. 3b delineating three clusters of parameters. To further characterize differences among ligand categories, we investigated whether the magnitude of parameters describing ligand response in each assay was different across the three clusters of ligands. To do so, we used a Kolmogorov-Smirnoff test to compare the distribution of parameter values in each cluster to that of the overall population (detailed in Supplementary Fig. 7). Only certain parameters in each cluster contributed to ligand discrimination, and they did so to different extents (Fig. 3c). Those in cluster A had the most weight, as $29.9 \%$ of comparisons identified at least one distribution of parameters significantly different from that of the whole population. Overall, $14.9 \%$ of comparisons in cluster B and 3.5\% in cluster $\mathrm{C}$ also significantly contributed to ligand discrimination. Alternatively, ordering parameters by type (Fig. $3 \mathrm{~d}$ ) revealed that efficacy-related parameters $\left(E_{\max }\right.$ and $\left.\tau\right)$ had the most weight in the classification $(52.0 \%$ and $36.0 \%$ of the comparisons, respectively, identified distributions different from the whole population) while $\log (\tau / \mathrm{KA})$ played a smaller role separating 

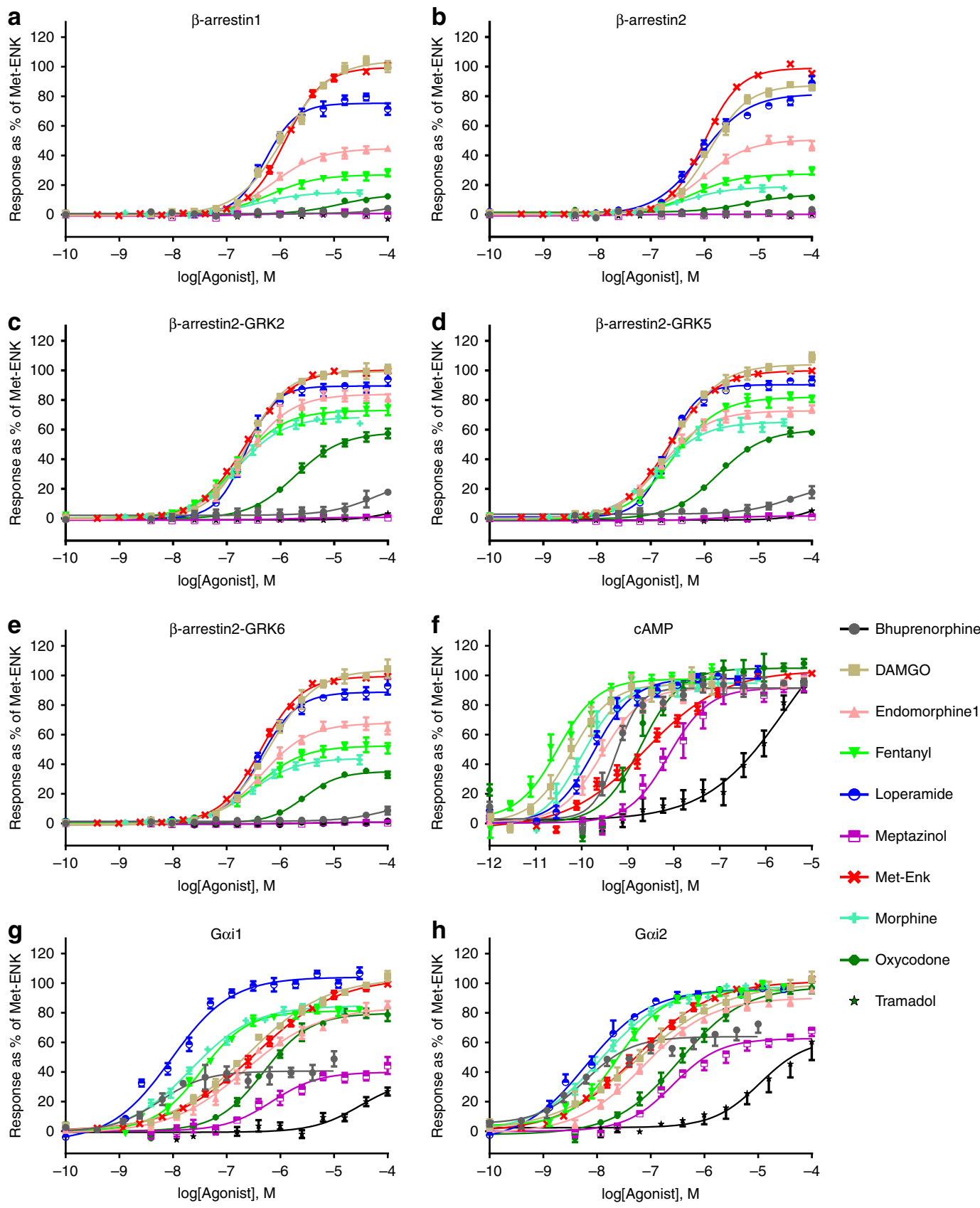

- Oxycodone

$\star$ Tramadol
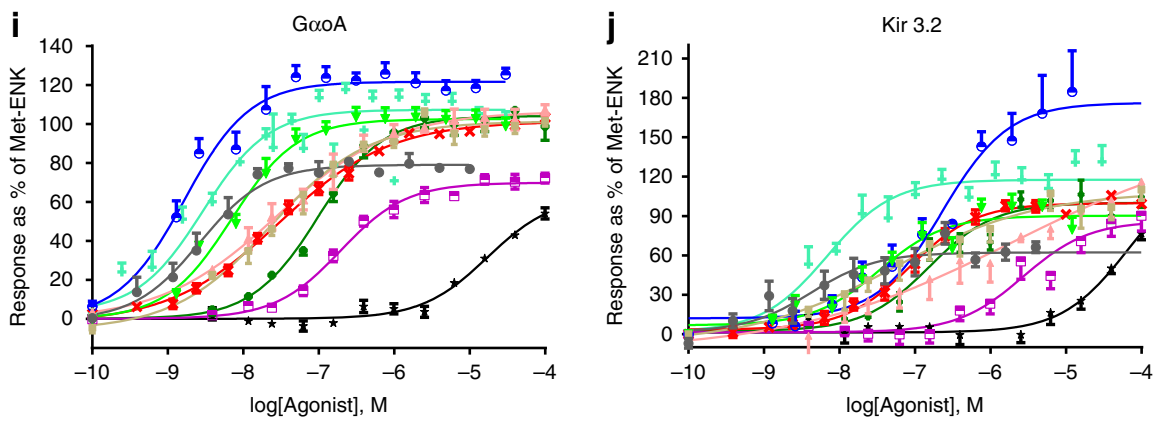

primary compound clusters (11.0\%). Finally, Supplementary Table 1 shows assay parameters significantly contributing to ligand clustering. The diversity of signals determining cluster assignment distinguishes this multidimensional classification from dichotomous comparisons underlying bias magnitudes.
Importantly, despite independence from bias magnitudes, the proposed classification strategy still allows to evaluate relative contributions of $\beta$ arr and $G$ protein signaling to ligand assignment to clusters. To access this information, drugs were reclustered using subsets of the data corresponding exclusively to $G$ 
Fig. 2 arr recruitment and G-protein responses generated by opioid ligands. Responses for prescription opioids and known hMOR ligands were monitored using BRET-based biosensors. The results correspond to mean \pm SEM of at least three independent experiments, normalized to the maximal effect of MetENK, which was tested in all experimental runs $(n=16-29)$. Curves were fit with the operational model and the logistic equation (the results from the

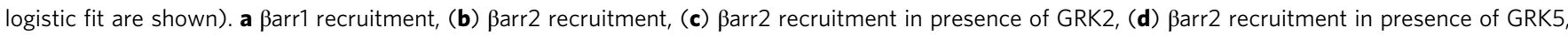
(e) Barr2 recruitment in presence of GRK6, (f) cAMP, (g) Goi1 activation, (h) Goi2 activation, (i) GooA activation, and (j) Kir3.1/3.2 activation. Net BRET values for Met-ENK are shown in Supplementary Fig. 5 and dose-response curves for all novel compounds appear in Supplementary Fig. 6. Operational and logistic parameters provided in Supplementary Data 1. Source data are provided as a source data file

protein (Supplementary Fig. 8a) or to ßarr (Supplementary Fig. $8 b)$ assays, and the resulting similarity matrix produced with each partial data set was compared with the matrix generated using the complete set of values. Differences between matrices were quantified as described in the Methods section and Supplementary Fig. 9, and expressed as the proportion of changes in ligand distances that were compatible with a switch in clusters between the two compared matrices. Clusters generated with $\beta$ arr data differed by only $11.5 \%$ from clusters produced with the complete data set (Fig. 3e), underscoring the similarity of drug classes defined by $\beta$ arr signaling patterns and complete signaling profiles. The partial data set for $G$ protein responses differed by $27.2 \%$ (Fig. 3e) from the complete ligand similarity matrix. Thus, although clusters generated with $\beta$ arr or with $G$ protein data sets resembled clusters produced with the complete data set more than did clusters generated with the corresponding randomized values, initial ligand clustering was more faithfully recreated by ßarr responses (Fig. 3e), indicating that that this signal was the one primarily driving classification in the complete matrix. Profiles graphically representing $E_{\max }$ and $\log (\tau / \mathrm{KA})$ values for $\mathrm{G}$ protein and $\beta$ arr readouts further highlight how the analysis clustered ligands according to type and magnitude of responses elicited (Fig. 4). Ligands in cluster \#3 were full, reasonably balanced agonists characterized by maximal effects at $\beta$ arr and $G$ protein readouts. Ligands in cluster \#2 were partial agonists for $G$ protein-mediated responses with measurable $\beta$ arr recruitment only in presence of overexpressed GRKs, while ligands in cluster \#1 displayed minimal or no $\beta$ arr recruitment and $G$ protein responses were overall smaller than in cluster $\# 2$.

While it may be feasible to monitor ten different signaling outcomes for a small group of ligands, it is unlikely that this could be done in high-throughput screening or structure-activity profiling. Hence, once we had identified the signals that contributed the most to drug classification, we determined whether a reduced number of assays could convey similar diversity. To this end, $\beta$ arr2 + GRK2, $\beta$ arr2 + GRK6, Gai2, and cAMP were chosen as respective prototypes of $\beta$ arr- and G protein responses. Similarity matrices generated from these individual signals or from their combinations were compared with the complete similarity matrix. As above, we compared each partial data set to the complete set of hMOR parameters, and then established if the proportion of ligands switching clusters was less than that observed for the corresponding randomized data set. For CAMP, the proportion of changes were larger than the expected random value (Fig. 3f), indicating minimal contribution of this signal to ligand classification. In contrast, for clusters generated with the other data subsets, the proportion of ligands switching clusters was significantly smaller than the random expectation (Fig. 3f), indicating that each of these signals significantly supported ligand discrimination in the complete data set, albeit to different extents.

The combination of $\beta$ arr2 + GRK2 and $\beta$ arr2 + GRK6 data was the best at reproducing clustering obtained with the complete hMOR similarity matrix (91.4\%; Fig. 3f). In comparison, the Gai2 data set either combined with cAMP or in isolation moderately recreated the clusters of the complete matrix (Gai2 $=74.4 \%$,
Gai2 + cAMP $=69.0 \%$ differences). Combining all four assays added little extra precision as compared with $\beta$ arr2 + GRK2 with ßarr2 + GRK6 (Fig. 3f). Thus, taken together, these data indicate that it is possible to first identify the signals that primarily contribute to signaling diversity of a group of compounds at a given receptor, and then use these signals as surrogate readout for screening campaigns over large collections of compounds.

Ligand clusters are informative of possible side effects. Preclinical studies suggest that signaling diversity of MOR agonists provides a means of improving tolerability of opioid analgesics $^{12-14}$. Therefore, it was of interest to determine if the pharmacodynamic clusters just defined could inform us about clinical side effects of ligands in each category. To address this issue, we first used standardized gamma (SD gamma) scores $^{33}$ to identify adverse events most frequently reported for opioids in the Food and Drug Administration's pharmacovigilance data base (Adverse Effects Report System (AERS)), and then calculated the scores of these events for each of the prescription opioids used in the study. These measures of side effect prevalence were associated to ligands in the different clusters by using the Euclidian distance between ligands in the similarity matrix. Tramadol was set as the arbitrary origin, and distances separating the rest of prescription opioids from tramadol in the $\log (\tau)-E_{\max }-\log (\tau /$ KA) matrix were consigned as measures of ligand similarity. These measures were then correlated to the SD gamma scores for each ligand's side effects. A complete list of the 80 events considered along with $r^{2}$ and $p$-values for each correlation is provided in Supplementary Data 2. Correlations that were significant $(p \leq$ $0.05)$ and/or explained at least $60 \%$ of the variance $\left(r^{2} \geq 0.60\right)$ were considered. Applying these criteria, ligand position in the $\log (\tau)-E_{\max }-\log (\tau / \mathrm{KA})$ matrix was correlated to 6 out of a total of 80 associations considered (7.5\%), including gastrointestinal (GI) events, respiratory depression, and somnolence (Table 1), all typically associated to opioid therapy ${ }^{34,35}$. These correlations confirm that signaling categories defined by unsupervised clustering can be associated to distinct frequency of report of undesired effects of opioid ligands.

$\log (\tau)$ and $E_{\max }$ were the main determinants of ligand position in the matrix constituting $89.0 \%$ of parameters effectively grouping ligands into clusters (Fig. 3d). Not surprisingly, if ligands were classified exclusively using these efficacy-related parameters, all side effects previously associated with ligand position in the $\log (\tau)-E_{\max }-\log (\tau / \mathrm{KA})$ matrix remained correlated with their positions in this efficacy-only matrix (Table 1). Actually, categories driven by efficacy measures associated with more side effects than clusters established by including $\log (\tau / \mathrm{KA})$ as an additional classification criterion (Table 1). Thus, even if functional affinity information within transduction coefficient affords better discrimination of ligands, it also acts as a confounder for cluster association to side effects.

Associating side effects to specific signals. Preclinical studies have suggested that MOR agonists that preferentially engage $G$ protein over $\beta$ arr responses could display less gastrointestinal and 
a

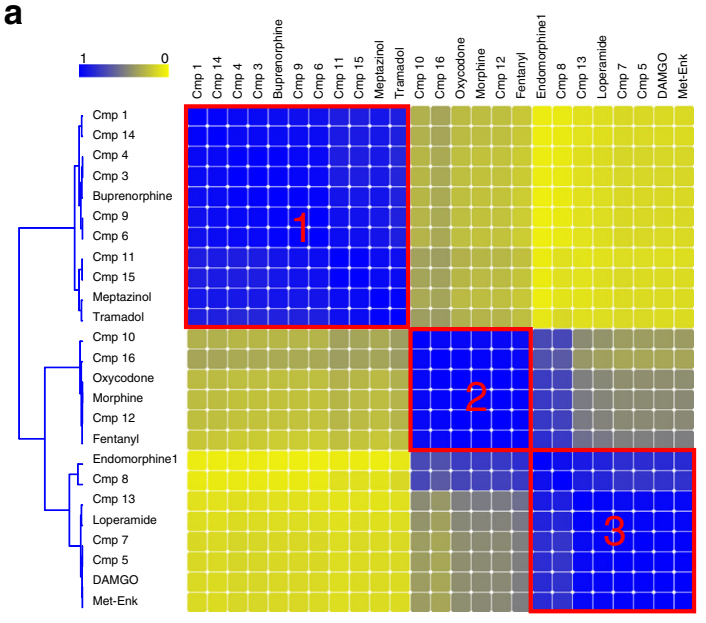

C

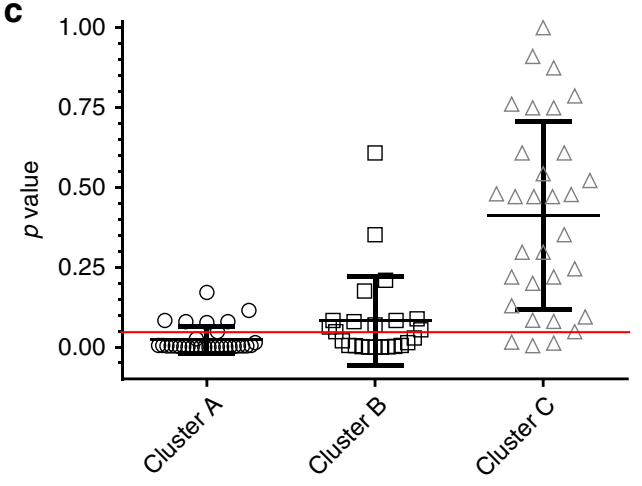

e

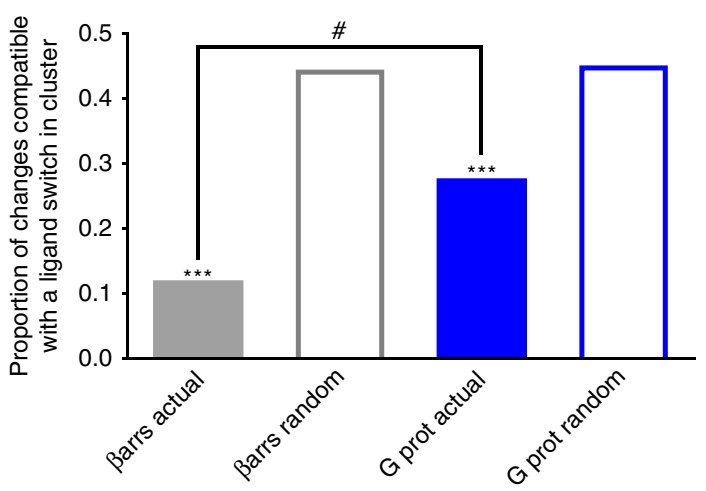

Vs complete hMOR data set b

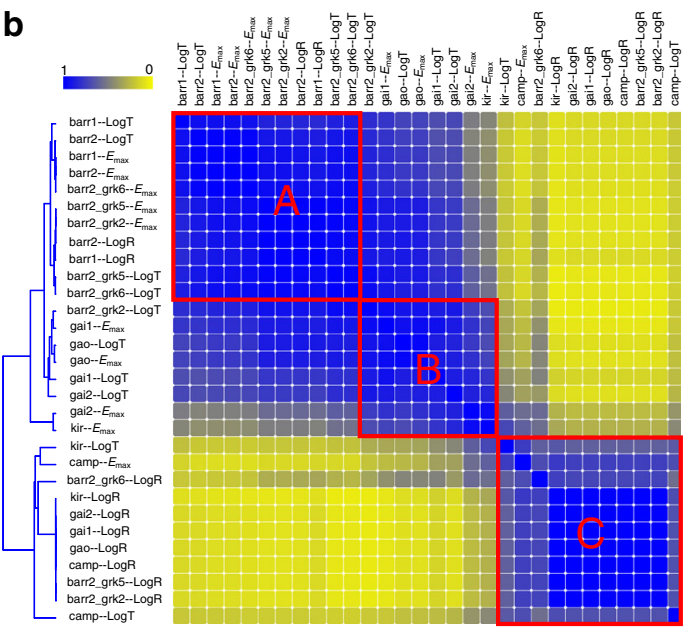

d

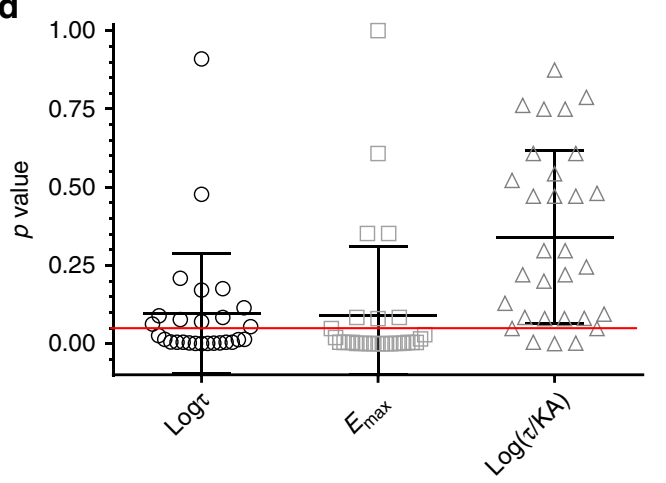

f

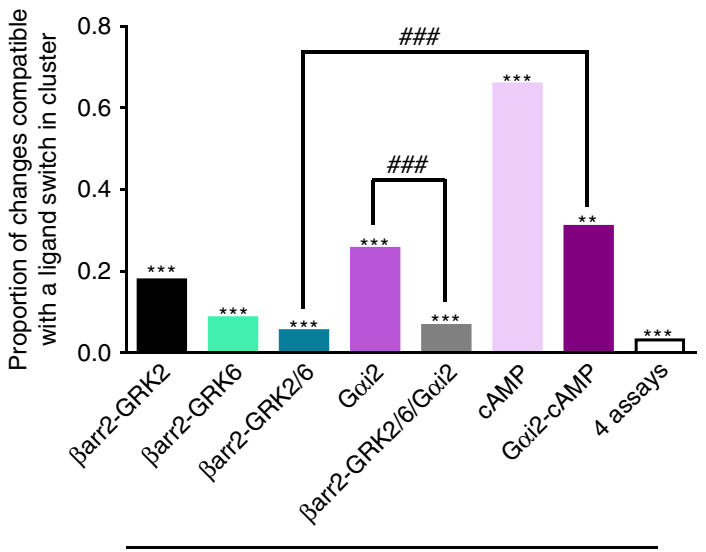

Vs complete hMOR data set

Fig. 3 Assignment of hMOR ligands into clusters is primarily driven by $\beta$ arr responses. Ligand (a) and parameter (b) similarity heatmaps for the complete hMOR data set. Yellow and blue, respectively indicate ligands/parameters that never or always cluster together. Distribution of parameters describing ligands within clusters shown in (a) was compared to their distribution in the whole population using a two-sample Kolmogorov-Smirnov test. Resulting $p$ values were plotted according to clusters shown in (b) (c) or to parameter type (d), mean \pm SD are also shown. Red line: $p=0.05$. Similarity matrices corresponding to partial data sets for $\beta$ arr- or G-protein-mediated responses were compared with the complete, reference hMOR data set. Filled bars: proportion of ligands changing clusters when comparing actual $\beta$ arr and G-protein data sets to the reference; empty bars: proportions observed by comparing simulations of random clustering to the reference data set. ${ }^{\star \star \star} p<0.001$; -zscore $\beta$ arr: $-5.375 ; z$-score $G$ protein: -6.092 . ${ }^{\#} p<0.05 ; z$-score difference: $-2.22(\mathbf{e})$. Similarity matrices generated for indicated partial responses were compared with the hMOR reference matrix. The results for actual data matrices are shown while results for random simulations were omitted. ${ }^{\star \star} p<0.01 ;{ }^{\star \star \star} p<0.001$ comparing partial data sets to their randomized controls; z-scores for comparisons between actual and randomized data: $\beta$ arr2-GRK2: -12.724; $\beta$ arr2-GRK6: -10.583; $\beta$ arr2-GRK2/GRK6:- 8.835; Goi2:

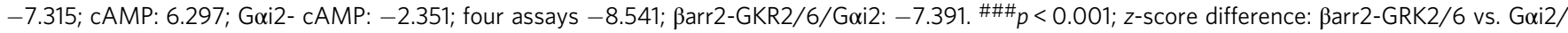
cAMP: -3.308; z-score difference: Goi2 vs Barr2-GRK2/6-Goi2: 3.754 (f). Source data provided in Supplementary Data 1 and as a source data file 


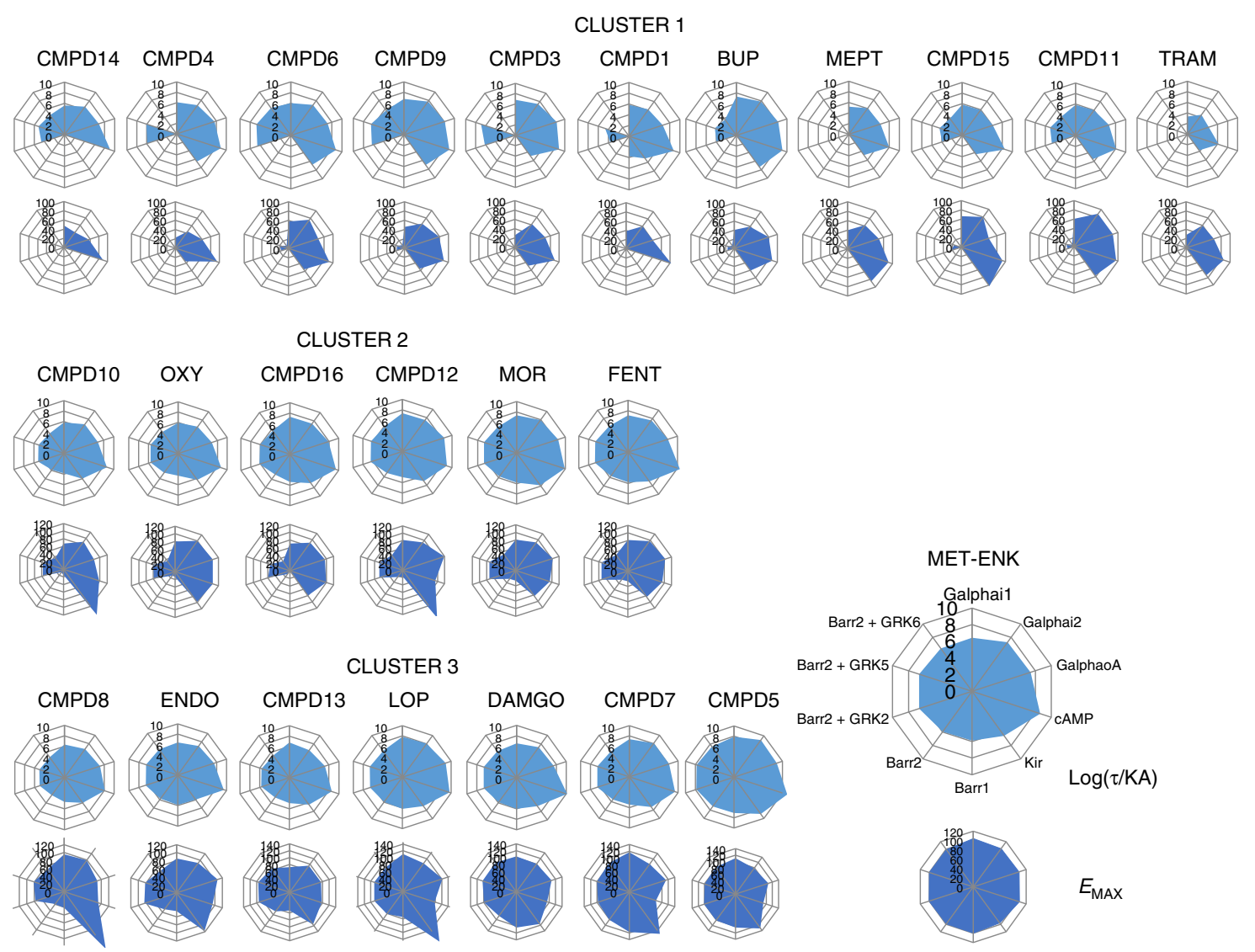

Fig. 4 Graphic representation of operational and logistic parameters for hMOR ligands populating different clusters. Operational transduction coefficients $(\log (\tau / K A))$ and logistic $E_{\max }$ values derived from concentration response curves generated by hMORs at ten different biosensors were represented as radial graphs. Each radius corresponds to the magnitude of $\log (\tau / K A)$ or $E_{\max }$ values. Transduction coefficients are in logarithmic scale, $E_{\max }$ values were normalized to maximal Met-ENK response, and are presented on linear scale. Source data provided in Supplementary Data 1

respiratory side effects in the clinic ${ }^{12-14}$. Hence, we were interested to find out whether AERS reports for opioids would distinctively correlate with ligand categories defined either by $G$ protein or $\beta$ arr signaling. There has been considerable debate as to whether biased signaling is best identified using $\log (\tau / \mathrm{KA})$ transduction coefficients ${ }^{17,24,25}$ or efficacy-related measures ${ }^{36,37}$. Hence, partial matrices in which drugs were classified according to $G$ protein or $\beta$ arr responses were generated using either Log $(\tau)-E_{\max }$ or $\log (\tau)-E_{\max }-\log (\tau / \mathrm{KA})$ as classification criteria. Supplementary Fig. 10 shows how frequency of faecaloma report correlates to similarity scores in these four partial matrices. Considering the classification based exclusively on $\log (\tau)-E_{\max }$ values, categories defined by $\beta$ arr and $G$ protein responses were both correlated to faecaloma report (Supplementary Fig. 10a), implying no differential association of these signals to the undesired event. In contrast, when $\log (\tau / \mathrm{KA})$ coefficients were additionally considered, faecaloma report correlated to $\beta$ arr, but not $\mathrm{G}$ protein responses (Supplementary Fig. 10b). However, it is worth considering how inclusion of $\log (\tau / \mathrm{KA})$ values breaks the correlation previously established with efficacy-based categories. BUP has a high transduction coefficient that cannot be distinguished from those of fentanyl (FEN) or loperamide (LOP), causing the partial agonist to move closer to these efficacious ligands in the matrix. Yet BUP's transduction coefficient is driven by its high affinity ${ }^{38,39}$, and regardless of its position among efficacious agonists its side effects profile remains determined by its partial efficacy, disrupting the correlation.

Opioid modulation of acute ileum contractility is G proteindriven by effectors that hyperpolarize myenteric neurons and inhibit neurotransmitter release by vagal terminals ${ }^{40-42}$, We used this $G$ protein-mediated response to ascertain that failure to correlate faecaloma report to categories partly defined by $\log (\tau /$ KA) was not due to the method itself. In effect, as shown in Supplementary Fig. 10c-e, frequency of faecaloma report correlated with $\log (\tau)$ but not $\log (\tau / \mathrm{KA})$ values describing ligand inhibition of ileum contraction.

Signaling and structural clusters convey complementary information about side effects. Structural criteria are used to classify, compare, and infer possible commonalities of in vivo responses for drug candidates ${ }^{43}$. We therefore compared categories of ligands defined by pharmacodynamic and structural criteria. Ligand structure was described using Tanimoto values ${ }^{44}$ derived from standard fingerprint representations (Supplementary Data 3-5), and these values were then clustered using the same NNMF/k-means method as previously applied on signaling profiles. The resulting clusters are shown in Supplementary Fig. 11a, and representatives of each structural group are provided in Supplementary Fig. 12. Structural and pharmacodynamic similarity matrices were then compared, indicating that $36.0 \%$ of changes in ligand distance were compatible with a switch in cluster when the two different criteria were applied. This value was significantly lower when compared with $43.5 \%$ switches observed using randomized structural data $(z$-score $=-2.803$; $p<0.01$ ), denoting some degree of statistical similarity between signaling and structural categories (Source data provided). However, the degree of similarity was low as schematically 


\section{Table 1 Pharmacodynamic and structural categories associate with frequency of report of undesired events for clinically} available hMOR ligands $\#$

\begin{tabular}{|c|c|c|c|c|}
\hline Undesired events associated with & Type of side effect & Preferred term & R square & p-value \\
\hline \multirow{6}{*}{$\begin{array}{l}\text { Functional categories defined by } \log (\tau)-E_{\max }-\log \\
(\tau / \mathrm{KA})\end{array}$} & Gastrointestinal/nutrition & Cachexia & 0.66 & 0.05 \\
\hline & & Faecaloma & 0.86 & 0.01 \\
\hline & & Gastrointestinal motility disorder & 0.81 & 0.01 \\
\hline & Respiratory events & Respiratory depression & 0.62 & 0.11 \\
\hline & & Respiratory rate decreased & 0.60 & 0.13 \\
\hline & Sleep disorder & Somnolence & 0.60 & 0.12 \\
\hline \multirow[t]{8}{*}{ Functional categories defined by $\log (\tau)-E_{\max }$} & Gastrointestinal/nutrition & Cachexia & 0.66 & 0.05 \\
\hline & & Faecaloma & 0.92 & 0.00 \\
\hline & & Gastrointestinal motility disorder & 0.62 & 0.06 \\
\hline & Respiratory events & Hypoventilation & 0.68 & 0.08 \\
\hline & & Oxygen saturation decreased & 0.61 & 0.12 \\
\hline & & Respiratory depression & 0.78 & 0.05 \\
\hline & & Respiratory rate decreased & 0.69 & 0.08 \\
\hline & Sleep disorder & Somnolence & 0.75 & 0.06 \\
\hline \multirow[t]{12}{*}{ Structural categories } & Respiratory events & Hypopnoea & 0.65 & 0.10 \\
\hline & & Yawning & 0.79 & 0.04 \\
\hline & Neuropsychiatric & Withdrawal syndrome & 0.74 & 0.06 \\
\hline & Change in drug response & Analgesic drug level increased & 0.70 & 0.08 \\
\hline & & Drug effect decreased & 0.64 & 0.10 \\
\hline & & Drug effect increased & 0.90 & 0.01 \\
\hline & & Therapeutic response increased & 0.89 & 0.02 \\
\hline & & Therapeutic response decreased & 0.74 & 0.06 \\
\hline & Pain & Breakthrough pain & 0.69 & 0.08 \\
\hline & & Complex regional pain syndrome & 0.64 & 0.11 \\
\hline & Itching & Pruritus generalized & 0.86 & 0.02 \\
\hline & Others & Therapy cessation & 0.76 & 0.05 \\
\hline
\end{tabular}

represented in Supplementary Fig. 11b. In keeping with this notion, clusters based on chemical structures were correlated with a different set of reported events than those associated with the pharmacodynamic clusters (Table 1). In particular, ligand distances in the matrix generated with chemical structures correlated with $12.5 \%$ of reported events, including pruritus, a typical opioid associated complaint ${ }^{45}$, as well as with reports of withdrawal and fluctuations in response and drug levels (Supplementary Data 6). Since structure determines pharmacokinetic properties, it is not surprising that structural categories associate with fluctuations in drug effects and even withdrawal symptoms ${ }^{46}$. On the other hand, and in spite of pharmacodynamic properties also being determined by structure, categories based on structural fingerprint representations failed to identify any of the events that associated with signaling categories, emphasizing the value of complementing structural information with a signal-based classification.

Ligand clusters generated with different GPCRs. We next examined whether clustering analysis could reveal pharmacodynamic similarities and differences among ligand responses generated at different opioid receptor subtypes. To do so, we used the same set of biosensors as for hMORs to monitor ligand activity at rat MORs (rMORs), human delta opioid receptor (hDORs) and rat DORs (rDORs). Corresponding input matrices containing logistic and operational parameters for each receptor (Supplementary Data 7-9) were analyzed as before to yield individual similarity matrices and associated heatmaps (Fig. 5a-c). Differences in clustering across receptor subtypes and species were evaluated by comparing similarity matrices for each receptor and are summarized in Fig. $5 d$. These comparisons revealed that the pattern of signaling diversity of this group of opioid ligands was reasonably conserved within the same receptor from different species. Indeed, in comparisons between rat and human MORs or rat and human DORs, the proportion of changes in ligand distances that were compatible with a switch in cluster was significantly less for actual as compared with randomized data sets (Fig. 5e), confirming congruent patterns across species.

In contrast, when clusters generated with data sets from MORs and DORs within the same species were compared, their differences were statistically indistinguishable from those obtained by comparing corresponding sets of randomized data (Fig. 5f), indicating that the analysis discerned the distinct pharmacological profiles of the two receptor subtypes. To identify the source of these differences, we compared the relative contribution of $\beta$ arr and $G$ protein responses in driving ligand clustering this time according to hDOR responses. Clusters generated with each partial data set bore statistical similarity to clustering done using the complete data set, and no statistical difference was revealed between clusters produced with $\beta$ arr and $\mathrm{G}$ protein parameters (Fig. $5 \mathrm{~g}$ ). To more precisely establish the weight of $\beta$ arr and Gprotein responses to clustering of ligands according to hDOR signaling, we investigated how every value in the similarity matrix changed when considering hDOR clusters produced with the complete data set, and clusters generated with pathway-specific data sets. As shown in Fig. 5h, the variations between the complete and the $G$ protein similarity matrices paralleled the differences between the complete and the $\beta$ arr similarity matrices, indicating the two types of signals similarly contributed to the classification of ligands according to responses generated at hDORs. In contrast, and consistent with the fact that ßarr recruitment was the main determinant in hMOR clustering, the differences between $G$ protein and complete matrices were 
a
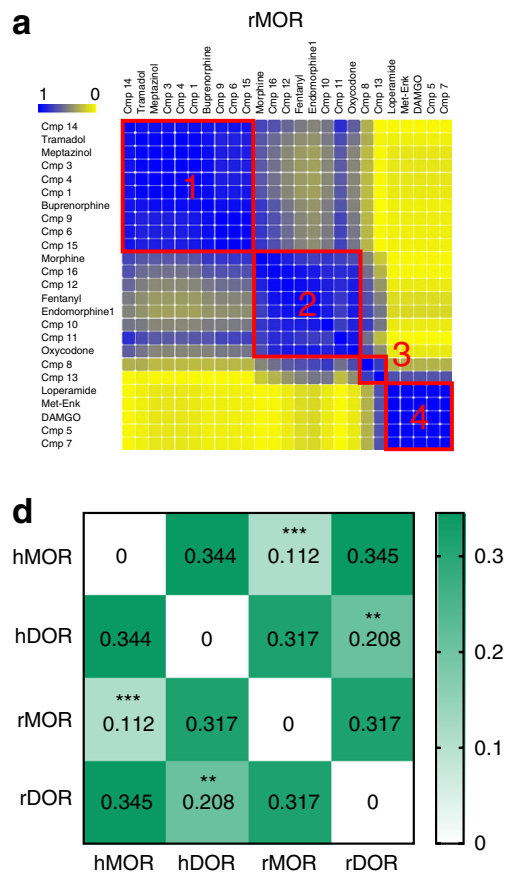

g

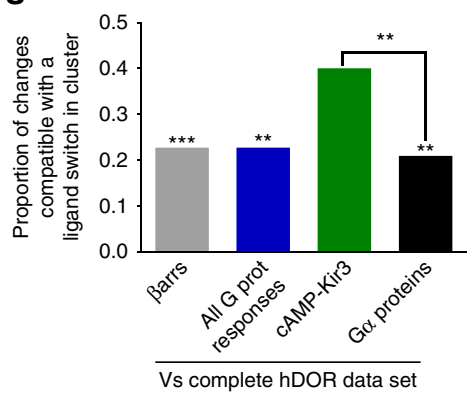

b

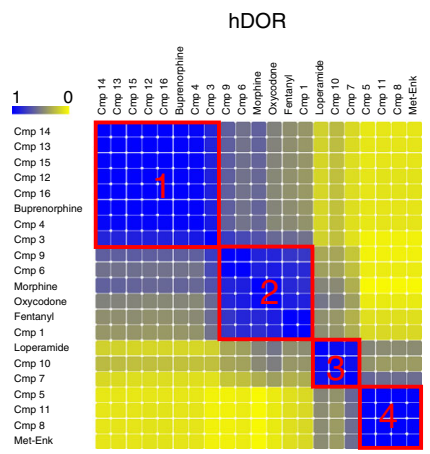

e

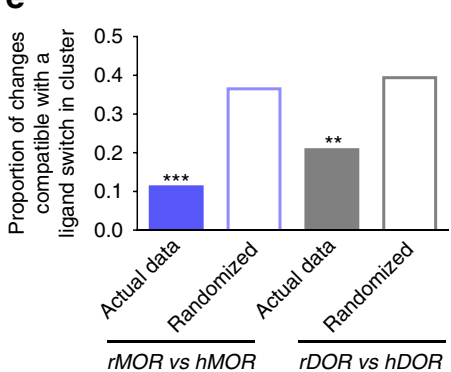

h

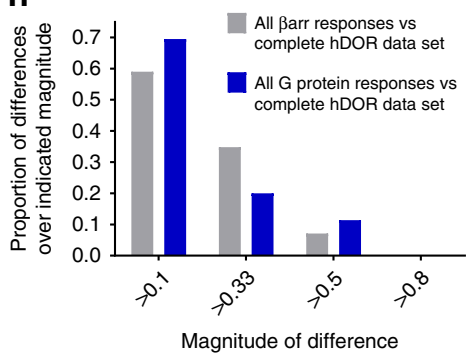

C

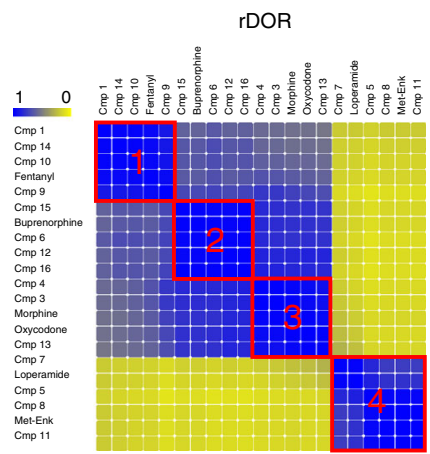

f

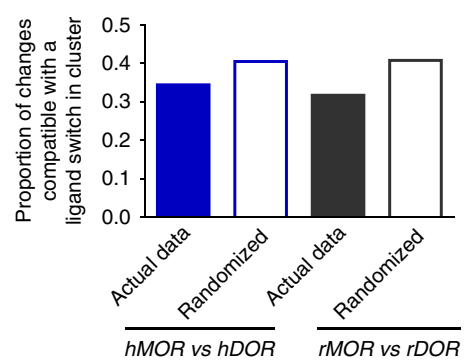

i

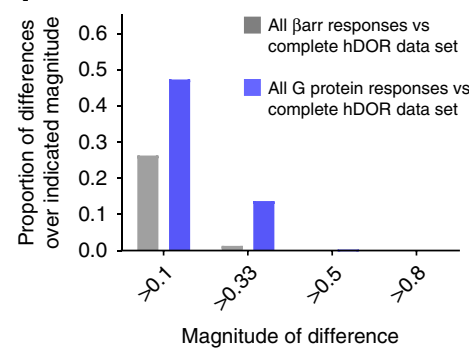

Fig. 5 Signaling profiles of opioid receptor ligands are conserved across species but not receptor subtypes. Ligand similarity heatmaps for rat MOR (a), human DOR (b), and rat DOR (c) data sets. Yellow and blue, respectively, indicate ligands/parameters that never or always cluster together. Proportion of ligands changing cluster for indicated comparisons; ${ }^{\star \star} p<0.01,{ }^{\star \star \star} p<0.001$ comparing actual to randomized data sets as in Fig. 3 (d). Similarity matrices for the same receptor compared across species. Filled and empty bars: proportion of ligands changing clusters when, respectively, comparing actual data and random clustering simulations to the reference matrix; ${ }^{\star \star} p<0.01$, ${ }^{\star \star \star} p<0.001$; $z$-score MOR subtypes: $-7.153 ; z$-score DOR subtypes: -2.742 (e). Similarity matrices for receptor subtypes within species compared as in (e). Actual and randomized data sets did not differ: $z$-score hMOR vs. hDOR: -1.502; z-score rMOR vs. rDOR subtypes: -1.567 (f). Similarity matrices for $\beta$ arr- or $\mathrm{G}$ protein-partial data sets were compared with the complete hDOR matrix as in (e); only comparisons for actual data are shown; ${ }^{\star \star} p<0.01,{ }^{\star \star \star} p<0.001$; $z$-scores versus corresponding randomized data: $\beta$ arr: -3.309 ; $G$ protein: -2.644; cAMP-Kir3.2: -0.309 , G $\alpha$ proteins: -3.286 . \#\# ${ }^{*}$ 0.01; $z$-score difference: cAMP-Kir3.2 vs. G $\alpha$ proteins: $2.515 . p=0.256 ; z$-score difference $\beta$ arrs vs. All G prot responses: $-0.656(\mathbf{g})$. A difference was calculated between every value $\mathrm{i}_{\mathrm{ij}}$ in the complete hDOR matrix and corresponding value $_{\mathrm{ij}}$ in indicated partial data sets. Histogram shows the fraction of differences with absolute value above indicated thresholds (h). Calculations described in (h) were repeated for the complete hMOR matrix and partial data sets for ßarr or G protein (i). Source data provided in Supplementary Data 7, 8, and 9 and source data files

more frequent and larger than those for the corresponding $\beta$ arr comparison (Fig. 5i). A graphical representation of hDOR clusters is given in Supplementary Fig. 13.

Finally, we assessed whether clustering according to signaling profiles could be extended to GPCRs that couple to effectors different than those activated by opioid receptors. For this purpose, we considered published ${ }^{47}$ and novel data generated with $\beta 2$-adrenergic receptor ( $\beta 2 \mathrm{AR}$ ) ligands including: (a) $\mathrm{G}$ protein-dependent responses (Gas activation, cAMP production, $\mathrm{Ca}^{2+}$ mobilization) $)^{48}$, (b) $\beta$ arr-mediated responses ( $\beta$ arr2 recruitment and receptor internalization), and (c) ERK signaling, a multifaceted response involving both $G$ proteins and $\beta$ arrs $^{49}$. Parameters describing concentration response curves for each of the readouts (Supplementary Data 10) were analyzed by NNMF and k-means to reveal four different drug categories (Fig. 6a). Cluster \#1, including isoproterenol (ISO) and norepinephrine (NE), was characterized by measurable agonist efficacy at all readouts. Salbutamol (SALB) and salmeterol (SALM) in cluster \#2 could be distinguished from the first category because of their minimal responses at $\beta$ arr-dependent readouts. Carvedilol (CARV) and propranolol (PRO) behaved as agonists only in the ERK pathway (Cluster \#3), while ICI118,555 and metoprolol (MET) had no efficacy except for inverse agonism at Gas and cAMP assays (cluster \#4). The complete signaling profiles for ligands in different clusters are provided in Supplementary Fig. 14.

As shown in Fig. 6b, partial data sets for Gas/cAMP/Ca ${ }^{2+}$ and for $\beta$ arr-recruitment/endocytosis recreated original clusters better 

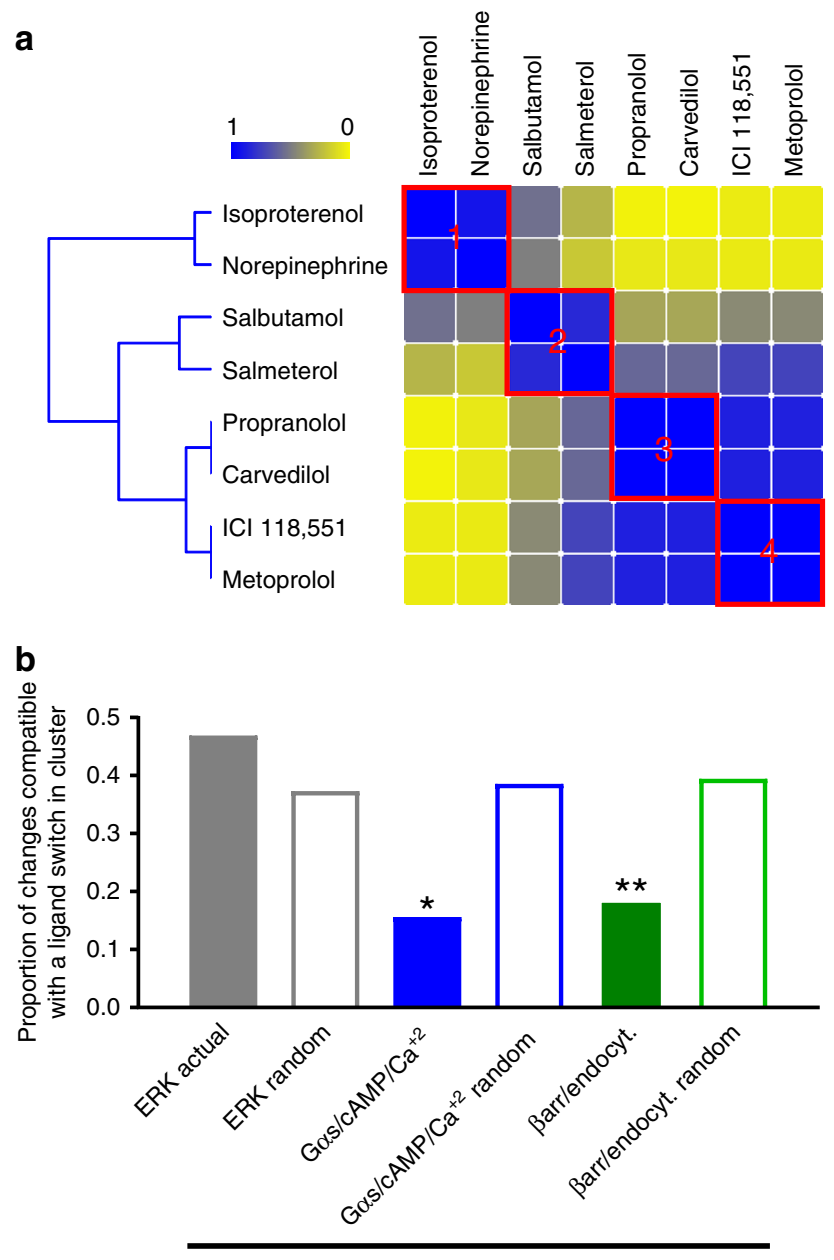

Vs complete $\beta 2 A D R$ data set

Fig. $6 \beta 2_{A D R}$ ligands cluster according to similarity in $\mathrm{G}$ protein and $\beta$ arrmediated responses. Ligand similarity heatmaps for $h \beta 2_{A D R s}$. Yellow and blue, respectively, indicate ligands/parameters that never or always cluster together. (a). Similarity matrices for partial data sets corresponding to ERK,

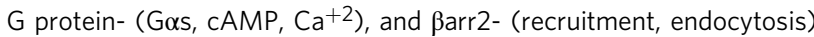
mediated responses were compared with the reference $\mathrm{h} \beta 2_{\mathrm{ADRs}}$ data set. Filled bars: proportion of ligands changing clusters when comparing actual ERK, G protein, and $\beta$ arr2 data sets to the reference; empty bars:

proportions observed comparing the reference data set to corresponding simulations of random clustering for partial matrices. ${ }^{\star} p<0.05,{ }^{\star \star} p<0.01$; $z$-score ERK: 1.523 ; $z$-score G protein: -2.446 and z-score $\beta$ arr: -2.636 (b). Source data provided in Supplementary Data 10 and source data files

than their corresponding randomized controls, indicating significant contribution of these signals to ligand clustering. To establish whether these categories were also relevant to human pharmacology, we evaluated their association to pharmacovigilance data. For this purpose, undesired cardiovascular and respiratory events most frequently reported for $\beta 2 \mathrm{AR}$ agonists and antagonists were first identified, and SD gamma scores representing the frequency with which these events were reported for the prescription ligands used in the study was correlated to their signaling similarity (measured as Euclidian distances in the full matrix) (Supplementary Data 11). We found that increasing distance from the agonist ISO was significantly correlated $(p<$ 0.05 ) with increasing frequency of reports for hypotension, decrease in blood pressure, sinus bradycardia, atrioventricular block, sinus arrest, and need for inhalation therapy (Table 2). Interestingly, the first four events in this list typically correspond to reduced sympathetic tone on cardiovascular function ${ }^{50}$. Hence, their more frequent association with ligands that clustered furthest apart from ISO is entirely consistent with gradual loss of efficacy at $\beta 2 \mathrm{AR}$. Moreover, these four events were also negatively correlated with ligand efficacy to induce Gas, cAMP, and $\mathrm{Ca}^{2+}$ signaling (Table 2), an overlap that is consistent both with the well-document role of these signals in maintaining heart chrono-, and inotropism ${ }^{51}$ and with the fact that $G$ proteindependent signals significantly drove ligand clustering. Gas, cAMP, and $\mathrm{Ca}^{2+}$ signaling categories also showed increasing reports of deleterious effects on respiratory function, some of them such as asthma, asthmatic crisis, status asthmaticus consistent with bronchoconstriction, as distance from ISO and $\beta 2 A R$ antagonism increased. Thus, pharmacodynamic categories defined by as few as eight ligands were sufficiently robust to reveal a well-known association between sympatholytic $\mathrm{CV}$ events or manifestations of bronchoconstriction and modulation of $G$ protein activity by $\beta 2 A R$ ligands. The observation reinforces the notion that unsupervised clustering of multidimensional signaling profiles allows the association of signals generated in simple cellular models to possible clinical effects of GPCR ligands.

\section{Discussion}

This study introduced a stepwise analysis in which GPCR ligands were organized into pharmacodynamic categories that could be then associated with clinically relevant responses. Pharmacodynamic parameters that best supported classification of ligands $\left(\left(\log (\tau)-E_{\max }-\log (\tau / \mathrm{KA})\right)\right.$ were empirically chosen by consecutively applying NNMF and k-means clustering to different combination of parameters informative of efficacies and functional affinities. The procedure was successfully applied to classify groups of ligands ranging from 8-320 in number, and representative of a multiplicity of signaling profiles.

Ligand categories generated with $\left(\log (\tau)-E_{\max }-\log (\tau / \mathrm{KA})\right)$ values from hMOR data were primarily driven by ligand diversity in ßarr signaling efficacy, but $G$ protein responses also contributed to the classification. Measured similarity among signaling profiles of prescription opioids present in the different categories was correlated with their corresponding frequencies of AERS reports for typical opioid side effects, indicating that the categories established by applying this exploratory method may allow to establish meaningful associations between in vitro signals and clinically relevant drug actions. This notion was further supported by observations that pharmacodynamic categories defined for $\beta 2 A R$ ligands were essentially driven by $G$ protein responses and associated to $G$ protein-driven sympatholytic effects ${ }^{52,53}$. Hence, by unveiling these well-documented associations, we established that clustering analysis of concentration-response parameters allows to associate multidimensional in vitro signaling profiles to clinical responses. Such use of curve parameters should prove beneficial for identifying signals that support specific responses of interest and for which mechanistic information is unavailable. Pharmacodynamic categories defined by efficacy-related parameters $\left(\log (\tau)-E_{\max }\right)$ had stronger and more frequent correlations to side effects than those defined by additional inclusion of affinity information provided by transduction coefficients. On the other hand, ligand differentiation was optimal when transduction coefficients were taken into account, calling for a discretionary decision on which parameters to use depending on the goal of the classification.

By classifying opioid ligands according to pathway-specific responses, it was possible to explore whether specific signals were driving typical side effects of opioids. We found that association of faecaloma report to categories defined by $G$ protein responses 
Table 2 Pharmacodynamic categories associate with frequency of report of undesired cardiovascular and respiratory events for clinically available $\beta 2_{\text {ADR }}$ ligands ${ }^{\#}$

\begin{tabular}{|c|c|c|c|c|}
\hline $\begin{array}{l}\text { Correlations between SD gamma scores for prescription } \beta 2 A D R \\
\text { ligands }{ }^{\star} \text { and }\end{array}$ & Type of side effect & Preferred term & R square & p-value \\
\hline \multirow[t]{6}{*}{ Functional categories defined by $\log (\tau)-\mathrm{E}_{\max }-\log (\tau / \mathrm{KA})$} & \multirow[t]{5}{*}{ CV } & Hypotension & 0.60 & 0.04 \\
\hline & & Sinus bradycardia & 0.58 & 0.05 \\
\hline & & Blood pressure decreased & 0.64 & 0.03 \\
\hline & & Atrioventricular block & 0.62 & 0.04 \\
\hline & & Sinus arrest & 0.68 & 0.02 \\
\hline & Respiratory & Inhalation therapy & 0.60 & 0.04 \\
\hline \multirow[t]{14}{*}{ Functional categories defined by Gs/cAMP/Ca2 + (partial matrix) } & \multirow[t]{8}{*}{ CV } & Hypotension & 0.80 & 0.01 \\
\hline & & Sinus bradycardia & 0.66 & 0.03 \\
\hline & & Blood pressure decreased & 0.81 & 0.01 \\
\hline & & Atrioventricular block & 0.72 & 0.02 \\
\hline & & Blood pressure systolic decreased & 0.64 & 0.03 \\
\hline & & Sinus arrest & 0.82 & 0.00 \\
\hline & & Ejection fraction decreased & 0.82 & 0.01 \\
\hline & & Heart rate decreased & 0.67 & 0.05 \\
\hline & \multirow[t]{6}{*}{ Respiratory } & Asthma & 0.64 & 0.03 \\
\hline & & Inhalation therapy & 0.59 & 0.05 \\
\hline & & Asthmatic crisis & 0.74 & 0.01 \\
\hline & & Status asthmaticus & 0.89 & 0.00 \\
\hline & & Throat irritation & 0.69 & 0.02 \\
\hline & & Choking & 0.64 & 0.03 \\
\hline
\end{tabular}

\#Only significant correlations were considered, full information in Supplementary Data 11. Similarity and SD gamma scores for Isoproterenol, norepinephrine, salbutamol, salmeterol, propranolol, carvedilol, and metroprolol were used to establish correlations. Source data provided in Supplementary Data 11 and source data files

was influenced by the parameters used in ligand classification. In $\log (\tau)-E_{\max }$ matrices, $G$ protein and $\beta$ arr categories were both directly correlated with reports for this side effect, implying that weaker agonists were associated with lower frequency of reports. In contrast, the correlation with $G$ protein responses was disrupted if $\log (\tau / \mathrm{KA})$ values were also considered, suggesting a scenario were $G$ protein signaling would not associate to side effects. This divergence as compared with $\log (\tau)-E_{\max }$ matrices is linked to the fact that despite its partial efficacy and a side effects profile consistent with partial agonism, $\log (\tau / \mathrm{KA})$ coefficients could not distinguish this low-efficacy-high-affinity ligand from much more efficacious agonists, such as morphine or oxycodone. In contrast to $\mathrm{G}$ proteins, $\beta$ arr categories correlated to faecaloma report independent of the parameters used for classification, as transduction coefficients for $\beta$ arr responses were consistent with BUP's low efficacy. $\log (\tau / \mathrm{KA})$ transduction coefficients are largely used to identify biased ligands ${ }^{17,25}$, so a word of caution is warranted for bias measures driven by functional affinity since only efficacy parameters are predictive of the magnitude of in vivo responses ${ }^{54}$.

It is also of interest that maximal responses for $\beta$ arr and $G$ proteins decreased in parallel across the different clusters, albeit not to the same extent. Indeed, $\beta$ arr signals gradually disappeared while those of $G$ proteins grew progressively smaller without completely vanishing. Such systematic imbalance between the two types of signals has been previously reported ${ }^{55}$, and is akin to system bias ${ }^{25}$ where $\beta$ arr responses are less well coupled to the receptor than $G$ protein signals. Within this context, absence of ßarr responses may simply indicate partial agonism and not signaling bias. As a matter of fact, all novel biased hMOR ligands presented herein as well as those published to date (i.e.: TRV130 ${ }^{12}$; PZM $21^{13}$ and Scripps compounds ${ }^{14}$ ) are partial agonists at $\mathrm{G}$ protein responses. This raises the possibility that currently available biased ligands could simply produce less side effects because they are partially effective at stimulating the receptor, and not necessarily because of greater efficacy for activating the $G$ protein over $\beta$ arr. A miss-interpretation of bias might be a problem for future clinical applications since a partial agonist may also produce a submaximal analgesic response. In this sense, it is of interest that $\beta$ arr-G protein signaling profiles of the latest hMOR ligands ${ }^{14}$ resemble those obtained in this study for BUP, a partially effective analgesic ${ }^{56}$. It is also worth considering that the clinical profile of TRV130, a partial hMOR agonist which was clinically tested as the first biased agonist for MORs, did not significantly differ from morphine's profile at doses with equivalent analgesic effects ${ }^{10}$. Finally, when PZM21 was independently tested after its initial description as biased agonist, it was shown to behave as a partial agonist in $\beta$ arr and $G$ protein readouts, and to produce respiratory depression commensurate with partial signaling ${ }^{10}$.

Structural similarity is another means for inferring common in vivo responses of therapeutic drug candidates early in the discovery process ${ }^{43}$. Here, when clusters established on the bases of signaling and structural resemblance were compared, they displayed nonrandom but marginal similarity. Different reasons could explain the low degree of similarity between categories established with structural and pharmacodynamic criteria, including incomplete representation of structural diversity of opioid ligands within the sample used, or different discriminatory power of signaling profiles and current descriptors of structural properties. Consistent with their low degree of similarity, structural, and pharmacodynamic categories were associated with different types of undesired events. Indeed, structural similarities were more frequently associated with fluctuations in therapeutic response, which are typically associated with pharmacokinetic properties $^{42}$. On the other hand, signaling categories specifically correlated with on-target side effects, pointing to the complementarity of both approaches when characterizing a limited number of compounds of interest.

In conclusion, we presented an unsupervised classification method that incorporates distinct and complementary data sources to comprehensively describe signaling diversity of GPCR ligands. The procedure identifies signaling imbalance independent of whether bias in the response co-varies with efficacy, it was applied to a large diversity of signaling profiles and distinguishes subtle differences in signaling preferences. 


\section{Methods}

Materials and reagents. Standard opioids were purchased from Cedarlane (Burlington, Canada) and Sigma-Aldrich (St. Louis, MO, USA). Fifteen novel compounds were provided by Pfizer Inc. (Worldwide Research and Development). (-)-Isoproterenol hydrochloride, (-)-norepinephrine, DL-propranolol hydrochloride, $( \pm)$ metoprolol $(+)$-tartrate salt, carvedilol, and salmeterol xinafoate were purchased from Sigma-Aldrich (St Louis, MO). ICI 118,551 and salbutamol hemisulfate were purchased from Tocris Bioscience (Ellisville, MO). Coelenterazine 400a was purchased from Biotium.

\section{Plasmids and DNA constructs. A cleavable signal sequence of influenza} hemagglutinin (MKTIIALSYIFCLVFA) and a Flag tag (MDYKDDDDA) were added to the human MOR1, rat MOR1, human DOR, and rat DOR and, their coding sequence optimized and synthetized as Strings DNA Fragments at GeneART (ThermoFisher Scientific). The DNA Strings were subcloned by Gibson assembly (New England Biolabs Canada) in pLVX-IRES-Puro (Clontech Laboratories, Inc). Untagged versions of the receptors were made by an internal NcoI deletion, removing the coding sequence of the Flag tag. Constructs encoding for GFP10-tagged receptors were made by PCR overlap; the coding sequence of each signal-peptide Flag-receptors was PCR-amplified to remove the stop codon and assembled by PCR overlap with the coding sequence of GFP10. The resulting PCR products were subcloned by Gibson assembly in pLVX-IRES-Puro. Constructs encoding the Epac-based cAMP sensor (GFP10-Epac-RlucII), RlucII-tagged Ga (ai1, $\alpha \mathrm{i} 2$, $\alpha \mathrm{oA}, \alpha s$ ), GFP10-G $\gamma 1$, GFP10-G $\gamma 2, \beta$-arrestin1-RlucII, $\beta$-arrestin2-RlucII, RlucII-G $\gamma 2$, human $\beta 2$-adrenergic receptor (h $\beta 2 A R$ ), and cmyc-h $\beta 2 A R-G F P 10$ were previously described (PMID: 15782186, PMID: 22534132, PMID: 23175530, PMID: 24309376, PMID: 19584306, PMID: 26658454, PMID: 16901982, PMID: 15155738). pCDNA3.1 ( + ) G $\beta 1$ was bought at Missouri University of Science and Technology (cdna.org). Plasmids encoding for the following proteins were generously provided as follows: GRK6 and GRK2 by Dr Antonio De Blasi (Istituto Neurologico Mediterraneo Neuromed, Pozzilli, Italy), GRK5 by Dr Robert Lefkowitz (Duke University, Durham, NC). Kir3.2-GFP10 by Dr Terry Hebert (McGill University, Montréal, Canada). Kir3.1 subunit by Dr. Deborah J. Nelson (University of Chicago, Chicago, IL).

Cell culture and transfection. HEK293 cells were a kind gift of Dr. Laporte, McGill University ${ }^{57}$. They were cultured in $100 \mathrm{~mm}$ Petri dishes (Sarstedt, Germany) at $37^{\circ} \mathrm{C}$ and $5 \% \mathrm{CO}_{2}$ in the Dulbecco's modified Eagle's medium (DMEM) supplemented with $10 \%$ fetal bovine serum, $2 \mathrm{~mm}$ L-glutamine and 100 unit $\mathrm{mL}^{-1}$ penicillin-streptomycin.

Transient transfections of vectors encoding BRET biosensors in combination with complementary signaling partners were performed in $100 \mathrm{~mm}$ Petri dishes $(3 \times$ $10^{6}$ cells) for G protein and Kir3.2 channel activation assays and in 96-wells culture plates coated with polyD-lysine (PerkinElmer, MA, USA) for $\beta$ arr recruitment assays $(32,000$ cells/well), using the polyethylenimine transfection reagent (Polysciences, PA, USA) at a PEI/DNA ratio of 3:158. For cAMP production assays, stable cell lines expressing the GFP10-Epac-RlucBRET2-cAMP biosensor ${ }^{59}$ were plated in six-wells plates (Greiner bio-one, Austria) and stably transfected with $1 \mu \mathrm{g}$ of either MORs or DORs (human or rat) biosensor using PEI. They were selected respectively using hygromycin $\left(100 \mu \mathrm{gL}^{-1}\right)$ and puromycin $\left(10 \mathrm{mg} \mathrm{mL}^{-1}\right)$.

BRET assays. Ligand preparation: Agonists were dissolved in DMSO and spotted on 96-well white bottom microplates (Greiner bio-one) using the HP D300 Digital Dispenser (Tecan Life Sciences). DMSO concentration was normalized for each point at $0.334 \%$.

Gai and Gao-activation assay: HEK 293 were co-transfected with DOR or MOR (human or rat), either of the BRET biosensors pairs: $\gamma 2$-GFP10/GaoA-99-RlucII (Ratio Receptor/GFP/RlucII: 1:0.6:0.12), $\gamma 2$-GFP10/Gai1-91-RlucII (Ratio Receptor/GFP/RlucII: 1:0.6:0.12), or $\gamma 2$-GFP10/Gai2-99-RlucII (Ratio Receptor/ GFP/RlucII: 1:0.72:0.12) together with untagged G $\beta 1$ (Ratio 1: 0.6$)^{26}$. Forty-eight hours after the transfection, the media was removed and the cells were washed with phosphate-buffered solution (PBS) then re-suspended in $\mathrm{PBS}+\mathrm{MgCl}_{2}(0.429 \mathrm{mM})$ at a protein concentration $\geq 0.6 \mu \mathrm{g} \mu \mathrm{L}^{-1}$. Coelenterazine 400 a was added to the cells to a final concentration of $5 \mu \mathrm{M}$ for $3 \mathrm{~min}$, and $100 \mu \mathrm{L}$ per well of this mix were subsequently distributed into the 96 -well-printed plates. Plates were read $5 \mathrm{~min}$ after on the Mithras LB 940 microplate reader (Berthold Technologies, Bad Wildbad, Germany), $3 \mathrm{~s}$ per well, with filters set at $400 \mathrm{~nm}$ (RlucII) and $515 \mathrm{~nm}$ (GFP10); BRET ratios were calculated as GFP10/RlucII emissions. Net BRET values were calculated by subtracting background BRET ratio observed in cells expressing donor $\mathrm{G}$ biosensors alone.

Gs activation assay: HEK293 cells stably expressing $\beta 2 \mathrm{AR}$ were transiently transfected with $200 \mathrm{ng}$ Gas-67-RlucII, $100 \mathrm{ng}$ G $\beta 1$, and $100 \mathrm{ng}$ GFP10-G $\gamma 1$. The day of the experiment, cells were washed with Hank's balanced salt solution (HBSS) (137 mM NaCl, $5.4 \mathrm{mM} \mathrm{KCl,} 0.25 \mathrm{mM} \mathrm{NaHPO}_{4}, 0.44 \mathrm{mM} \mathrm{KH}_{2} \mathrm{PO}_{4}, 1.8$ $\mathrm{mM} \mathrm{CaCl}_{2}, 0.8 \mathrm{mM} \mathrm{MgSO}_{4}, 4.2 \mathrm{mM} \mathrm{NaHCO}_{3}, \mathrm{pH} 7.4$ ) supplemented with $0.1 \%$ glucose and $0.1 \%$ BSA. Coelenterazine $400 \mathrm{a}$ (Coel-400a, Biotium) was added for $5 \mathrm{~min}$ to the wells $(2.5 \mu \mathrm{M})$, then $\beta$-adrenergic compounds were added for $4.5 \mathrm{~min}$. BRET was measured and calculated as described above.
Kir 3.2 channel activation assay: HEK 293 were plated onto $100 \mathrm{~mm}$ Petri dish and transfected with DOR or MOR (human or rat), the Kir3.2-GFP10/ $/ 2$-LucII BRET biosensor pair together with untagged Kir3 channel and $G$ protein subunits $^{27}$ at a ratio of 1:1:0.075:1:0.5, respectively. The BRET assay was performed as described above.

$\beta$-arrestin recruitment: HEK 293 cells were co-transfected with sp-FLAG-DORGFP10 or sp-FLAG -MOR-GFP10 (human or rat) and $\beta$ arr1/2-RlucII for $\beta$ arrestin1/2 recruitment at a ratio receptor/construct of 1:0.06. Recruitment of $\beta$ arr2-RlucII was also tested in the presence of, GRK2, GRK5, GRK6 (Ratio receptor/GRK DNA: 1:0.1). Forty-eight hours after transfection, cells were washed with PBS then incubated in Tyrode's solution $(140 \mathrm{mM} \mathrm{NaCl}, 2.7 \mathrm{mM} \mathrm{KCl}, 1 \mathrm{mM}$ $\mathrm{CaCl}_{2}, 12 \mathrm{mM} \mathrm{NaHCO}_{3}, 5.6 \mathrm{mM}$ D-glucose, $0.49 \mathrm{mM} \mathrm{MgCl}_{2}, 0.37 \mathrm{mM} \mathrm{NaH}_{2} \mathrm{PO}_{4}$, $25 \mathrm{mM}$ HEPES, $\mathrm{pH} 7.4$ ) for $30-60 \mathrm{~min}$ at $37^{\circ} \mathrm{C}$. Indicated concentrations of agonists, diluted in Tyrode buffer, were added to the wells for $10 \mathrm{~min}$, then cells were incubated for $5 \mathrm{~min}$ with Coelenterazine $400 \mathrm{a}(2.5 \mu \mathrm{M})$. BRET2 readings were taken at $37^{\circ} \mathrm{C}$ as detailed above. For $\beta 2 \mathrm{AR} \beta$-arrestin 2 recruitment, HEK cells were transiently transfected with $50 \mathrm{ng} \beta$ arr2-RLucII and $300 \mathrm{ng} \beta 2 \mathrm{AR}-\mathrm{GFP} 10$. The day of the experiment, cells were washed with HBSS supplemented with $0.1 \%$ glucose and $0.1 \%$ BSA. $\beta$-adrenergic compounds were added to the wells for $10 \mathrm{~min}$, then coelenterazine 400 a was added for $5 \mathrm{~min}$ to the wells $(2.5 \mu \mathrm{M})$. BRET was measured and calculated as described above.

cAMP production assay: Stably-transfected cells expressing the GFP10-EpacRlucBRET2-cAMP biosensor ${ }^{59}$ and either MORs or DORs were seeded at a density of 30,000 cells/well in a high glucose medium supplemented with $10 \%$ newborn calf serum, and grown on 96-well polylysine-coated plates for $48 \mathrm{~h}$. Cells were later transferred to Tyrode buffer and incubated for $15 \mathrm{~min}$ at $37^{\circ} \mathrm{C}$. Coelenterazine $400 \mathrm{a}$ was then added to a final concentration of $5 \mu \mathrm{M}$. Five min later, forskolin (Bioshop, Canada) was introduced (final concentration: $10 \mu \mathrm{M}$ for rMOR, $15 \mu \mathrm{M}$ for $\mathrm{rDOR}$, and $25 \mu \mathrm{M}$ for hMOR and hDOR) followed, 3.5 min later, by increasing concentrations of ligands. BRET2 readings were taken $5 \mathrm{~min}$ after ligands were introduced $^{28}$

Guinea pig ileum assays. Male Hartley guinea pigs were anesthetized using isoflurane followed by exsanguination. The myenteric plexus of the ileum was dissected according to the method described by Cowie $\&$ al. ${ }^{41}$. Briefly, a portion of the ileum was removed $(10 \mathrm{~cm}$ distal to the cecum) into which a glass rod was inserted. The myenteric plexus was removed from the circular longitudinal muscle via gentle scraping with a moist cotton swab and separated from the muscle using forceps. The resulting myenteric tissue was cut into $2.5 \mathrm{~mm}$ strips and placed in oxygenated Krebs buffer $\left(37^{\circ} \mathrm{C}\right.$, gassed with $\left.95 \% \mathrm{O}_{2} / 5 \% \mathrm{CO}_{2}\right)$ and tensioned to a baseline tension of $2000 \mathrm{mg}$. The tissues were washed, equilibrated for $30 \mathrm{~min}$, and subsequently tested for viability with a maximal concentration of Carbachol $(300 \mathrm{nM}$, three times with $10 \mathrm{~min}$ of washing, and $10 \mathrm{~min}$ of equilibrating in between additions). The final prime was followed by a $20 \mathrm{~min}$ wash period followed by a 20 min equilibration period before the start of the experiment. Tissues were continually stimulated with $0.1 \mathrm{Hertz}$ for $1 \mathrm{~ms}$ at 20 volts (producing a stimulation equivalent to $80 \%$ of the maximal contractile response). Following a $10 \mathrm{~min}$ baseline stimulation period, the kappa opioid antagonist nor-binaltorphimine was added ( $5 \mathrm{nM}$ final) and incubated for $10 \mathrm{~min}$. Finally, cumulative concentration-response curves were generated to each test ligand or vehicle control (DMSO). Isometric tension data (in $\mathrm{mg}$ ) were collected.

All procedures performed on these animals were in accordance with regulations and established guidelines and were reviewed and approved by Pfizer Institutional Animal Care and Use Committee.

Curve fitting. Concentration response curves describing ligand responses by different receptors (hMOR, hDOR, rMOR, rDOR, and hb2ADR) were analyzed with Graphpad Prism6, using built-in 3 or 4 parameter logistic equations to obtain independent pEC50 and $E_{\max }$ values for each receptor-biosensor pair:

$$
y=a+(b-a) /\left(1+10^{(\log E C 50-x) * c}\right)
$$

( $y \rightarrow$ measured response; $a \rightarrow$ minimal asymptote, $b \rightarrow$ maximal asymptote; $b-a$ $\rightarrow E_{\max } ; \mathrm{c} \rightarrow$ slope)

Concentration response curves were additionally analyzed with the operational model of Black and Leff ${ }^{32}$. As above, curves representing responses elicited by the same receptor at each of the ten different biosensors were fit independently. Fitting was done using Graphpad Prism6 after introducing a set of equations kindly provided by Dr Christopoulos:

$A=10^{x}$

operate $1=$

$\left((1+A) /\left(10^{\log R} * A\right)\right)^{n}$

(used to fit full agonists)

$$
\begin{aligned}
& \text { operate } 2= \\
& \begin{array}{l}
\left(\left(1+A / 10^{\log K A}\right) /\left(10^{\log R} * A\right)\right)^{n} \\
\quad \text { (used to fit partial agonists) }
\end{array}
\end{aligned}
$$




$$
\begin{aligned}
& \text { Full agonist }= \\
& \text { basal }+\left(E_{\max }-\text { basal }\right) /(1+\text { operate } 1) \\
& \text { Partial agonist }= \\
& \text { basal }+\left(E_{\max }-\text { basal }\right) /(1+\text { operate } 2)
\end{aligned}
$$

basal $\rightarrow$ response observed in the absence of agonist; $E \mathrm{max} \rightarrow$ maximal response of the system; $n \rightarrow$ slope of the function which links occupancy to response; $K A \rightarrow$ functional affinity (partial agonists); $\log (\mathrm{R}) \rightarrow \log (\tau / \mathrm{KA})$.

When using the logistic model, the fits for three and four parameter curves were compared and the best fit taken. If no fitting was possible without constraints, the minimal asymptote was fixed to zero; if this was unsuccessful, the Hill coefficient was additionally fixed to one (i.e.,: only the three parameter fit was considered). If both these constraints proved unsuccessful, and in curves with no inflection point for maximal effect, the highest experimental value was considered $E_{\max }$. The latter procedure forced the maximal response of very weak partial agonists within the range of experimental data avoiding aberrant predictions due to extrapolation. If no fitting was possible following these constraints, no fitting (NF) status was consigned. If fitting was possible, we made sure that all curves had a Span $>3 \mathrm{x}$ SEM, otherwise they were considered as no response (NR).

As used in this study, the operational model does not yield $\log (\tau)$ or pKA values for full agonists ${ }^{25}$, which were consigned as not available (NA). In these circumstances, $E_{\max }$ values were used to differentiate these compounds from partial agonists, and differences among full agonists were established through their consolidated $\log (\tau / \mathrm{KA})$ coefficients. It should also be noted that by independently fitting curves for different biosensors, the model does not contemplate interconversion among distinct receptor states stabilized by different effectors.

\section{Feature reduction, and ligand clustering.}

1. Each receptor was represented by a matrix composed of 25 ligands (21 for DORs $) \times 30$ parameters $\left(E_{\max }, \log (\tau)\right.$ and $\log (\tau / \mathrm{KA})$ for ten assays). This matrix was created by sampling from the normal distribution around each parameter using the mean and standard deviation thereby incorporating the variance associated with each data point and propagating it through the clustering method. In order to correct for scale differences between parameters, we standardized each column to range between 0 and 1 according to:

$$
\text { Standardized value }=\frac{X_{i j}-\text { minimum }_{j}}{\text { maximum }_{j}-\text { minimum }_{j}}
$$

for every ligand $i$ and every parameter $j$.

2. Process (1) was repeated 1000 times to create 1000 data matrices each independently put through the following procedure (Supplementary Fig. 2).

3. Nonnegative matrix factorization (NNMF) was used to reduce dimensionality of the data and create the $\mathrm{W}$ (ligand ${ }^{*} \mathrm{k}$ ) and $\mathrm{H}\left(\mathrm{k}{ }^{*}\right.$ parameter) basis vectors thereby removing noise and redundancy. We used sparse NNMF to ignore missing data ("NA", "NF", and "NR" curves). Difference between the original matrix $\mathrm{V}$ and the product of $\mathrm{W}^{*} \mathrm{H}$ was minimized to less than 1e-7.

4. K-means clustering was performed on the $\mathrm{W}$ basis vector, where the number of clusters equals the number of basis vectors from $\operatorname{NNMF}(K=k)$, to assign each compound into a cluster. Note: the phenotypic parameter clusters were obtained using the $\mathrm{H}$ vector instead of the $\mathrm{W}$ one.

5. Steps 3 and 4 were repeated 250 times to quantify the fraction of times each compound clustered together resulting in a ligand ${ }^{*}$ ligand frequency matrix ranging from 1 (always clustered together) to 0 (never clustered together). This iterative process quantifies both global and local minima/maxima arising from small variances in clustering resulting from the randomized starting vectors for NNMF and k-means.

6. (a) The entire process including feature reduction and clustering (3-5) was repeated for different values of $\mathrm{k}(\mathrm{k}=2$ to $\mathrm{k}=7)$, providing a frequency matrix for each $\mathrm{k}$. (b) These six frequency matrices were averaged together to quantify ligand similarity independent of the number of features used as each $\mathrm{K}$ may extract unique patterns that may be complementary or orthogonal to results from different K's.

7. Steps 3-6 were independently performed on each of the 1000 sampled data matrices providing 1000 composite similarity matrices.

8. These 1000 matrices were averaged to create a final frequency matrix quantifying how often ligands clustered together and representing total ligand similarity across all concentration response curves.

9. We visualized the similarity matrix using a dendrogram and a heat map using Orange, created from the distance between each compound in the similarity matrix using a Pearson Correlation.

Simulation of virtual compounds. We built 16 profiles showing bias and various potencies/efficacies by selecting ranges of KA- $\tau$ pairs across six imaginary biosensors. So that our virtual compounds respect these ranges, we invented them by sampling random values of KA and $\tau$ within the bounds associated to the imaginary biosensors specified per profile. We used this procedure 20 times for each profile yielding 320 virtual ligands.

As for curve fitting, simulations were conducted under the assumption of independence across biosensors. Using the operational model equation, we generated corresponding concentration response curves (CRC) to which we added $10 \%$ noise using the flat distribution. Noisy CRCs were then fitted to both the logistic equation and the operational model equation using the Bayesian inference engine STAN ${ }^{60}$ to yield values of $E_{\max }, \mathrm{pEC} 50$, $\tau$, and KA and their associated distributions (from which we computed a standard error of the mean to use in the NNMF pipeline) (values in Supplementary Data 12). The best estimate for $\tau / \mathrm{KA}$ ratio and its distribution of draws were also computed directly within the fitting process as a transformed parameter.

Selections of subsets of parameter estimations and associated SEM were used in NNMF/k-means clustering. The resulting matrices of frequency of co-occurrences were used to compute distance metrics, hierarchical clustering trees, and tSNE plots whose leaves and data points were colored by profile (Fig. 1, Supplementary Fig. 3, Supplementary Fig. 4).

Clustering of pharmacological parameters. The 25 ( 1 per ligand) values for each parameter array $(\mathrm{P})$ were distributed into four smaller arrays corresponding the ligand clusters. We then utilized a two-sample Kolmogorov-Smirnov test to compare each sub-array to the original array $(\mathrm{P})$ to measure if these were randomly sampled from the original array. This provides four $p$-values for each parameter. A significant $p$-value indicates ligands in that cluster are biased toward a specific response for that parameter. This process was repeated for each of the 30 parameters. These $p$-values were then sorted according to (i) the type of parameter considered (e.g., pEC50, $E_{\max }$, or $\log (\tau / \mathrm{KA})$ ) or (ii) measurement similarity acquired from the similarity matrix obtained by the NNMF/k-means method detailed above using the $\mathrm{H}$ basis vector instead of the $\mathrm{W}$. The procedure is summarized in Supplementary Fig. 7.

Comparing clusters among complete data sets. To compare clustering similarity between two different data sets we implemented two approaches: (a) Directly comparing the two similarity matrices using pairwise differences and (b) quantifying the overall difference between the two matrices using random simulation to obtain a difference threshold and to establish significance.

Direct comparison: We calculated the difference between every paired value in similarity matrix A and B (representing the similarity between compounds $\mathrm{i}$ and $\mathrm{j}$ ):

$$
\text { Difference }=A_{i j}-B_{i j}
$$

The resulting difference matrix is of equal dimensionality to $\mathrm{A}$ and $\mathrm{B}$, ranging from 1 (compounds $\mathrm{i}$ and $\mathrm{j}$ are always clustered together in $\mathrm{A}$ but never in $\mathrm{B}$ ) to -1 (always in B but never in A).

Thresholding and random simulation: We compared the difference in Euclidian distance for every pair of ligands $i$ and $j$ between similarity matrices for data set $\mathrm{A}$ and $\mathrm{B}$ :

$$
\text { Difference }=\left\|L_{i A}-L_{j A}\right\|-\left\|L_{i B}-L_{j B}\right\|
$$

Where $\mathrm{L}_{\mathrm{iA}}$ and $\mathrm{L}_{j \mathrm{~A}}$ are row vectors representing the similarity of $\mathrm{L}_{\mathrm{i}}$ and $\mathrm{L}_{\mathrm{j}}$ to all other ligands in matrix A. We then used random clustering replicates (detailed below) to identify a cutoff value to determine which difference values corresponded to a significant variation between A and B. The final comparison between data set $\mathrm{A}$ and $\mathrm{B}$ was represented as a proportion:

$$
\text { Fraction change }=\frac{\# \text { significant differences }}{\text { Total\# of comparisons }}
$$

Only ligands tested in both data sets were used (e.g., comparing hMOR to hDOR only used the 21 shared ligands).

Random clustering: For each data set, we created 50 random input data matrices by permuting all mean-standard deviation pairs of data points within the original data matrix. Each random matrix was therefore specific to and equal in size and shape to the original data (e.g. hMOR: $(25 * 30)$; hMOR- $\beta$ arr: $(25 * 15))$. We then repeated the entire NNMF/k-means clustering method on each data-shuffled random matrix resulting in 50 random clustering frequency matrices for each data type.

To determine a cutoff value representing significant variation between any two data sets (Difference threshold), we calculated the Euclidian Distance between pairs of compounds in the same cluster and compounds in different clusters for each of the 50 trials. The threshold is the mean value of the overlap between the "same cluster" distribution and the "different cluster" distribution (see Supplementary Fig. 9). Threshold values range between 0.95 and 1.5. Using these thresholds, it was possible to calculate the proportion of significant variation between two matrices To quantify if this change was significant, we calculated the fraction of significant changes (using thresholds) between the clustering from the 50 randomized data sets (e.g., 50 random hDOR) compared with reference cluster (e.g., hMOR). The resulting distribution of 50 values represented the proportion of random changes from the reference. This distribution was used to calculate a $z$-score for the 
difference value of the actual data (hMOR vs hDOR):

$$
\mathrm{z} \text {-score }=\frac{(\text { fraction change in actual data })-(\text { mean random change })}{(\text { STD random changes })}
$$

\section{Comparing clusters generated with complete data and subsets. In order to} calculate whether clustering from data subset I (e.g., hMOR-Barr) changed more than data subset J (e.g., hMOR-G protein) compared with the complete data set clusters (e.g., hMOR), we compared the 50 random similarity matrices to the reference (e.g., hMOR) and calculated the fraction of significant changes using the method detailed above. As a result, we obtained an array of 50 values representing the random change from reference. This array was created for both subsets (e.g., hMOR- $\beta$ arr and hMOR-G protein). We then iteratively, with replacement, randomly sampled 1 value from each of these arrays and calculated the difference to create a distribution of 1000 values indicating the random expected difference between these two subsets of data. We then calculated a $z$-score using the mean and standard deviation of this distribution and the actual observed difference.

Clustering ligands according to structural similarities. Each ligand was represented using three standard fingerprint representations: (ECFP-6) ExtendedConnectivity Fingerprints (ECFPs) (http://accelrys.com/products/collaborativescience/biovia-pipeline-pilot/), Functional-Class Fingerprints (FCFPs) and MDL MACCS keys. A similarity matrices for each different fingerprint was generated for the 25 ligands in the data set, where each value in the matrix $\left(\mathrm{S}_{\mathrm{i}, \mathrm{j}}\right)$ corresponds to the Tanimoto similarity value between compound $i$ and compound $j$ and ranges from a value 0 to 1 ( 1 being most similar). We combined these three matrices into a single matrix of dimensions ( 25 compounds $\times 75$ comparisons), and repeated the NNMF/ k-means clustering algorithm on the data to yield a structural similarity matrix.

Correlating signaling data to side effect report frequency. A list of all MORactive compounds was created by searching DrugBank for all approved drugs which activate MOR. The resulting list was intersected with the list of drugs in the FDA's Adverse Event Reporting System data for which a standardized gamma (SD gamma) score could be generated at the preferred term (PT) level according to the method of Johnson et al. ${ }^{33}$. Briefly, SD gamma scoring is a statistical approach to identify disproportionately high, or low, numbers of drug-event occurrences by normalizing for number of drugs and number of event reports. SD gamma scores for each event were averaged for all resulting MOR compounds, and PT events were sorted by average score to produce a listing of high-scoring events most clinically relevant to opioid therapy ( 80 highest scores were considered). A similar procedure was completed to find the 80 side effects associated with $\beta 2 \mathrm{ADR}$-active compounds.

Individual drug SD gamma scores for frequently reported events were then correlated to Euclidian distances separating prescription opioids (tramadol, buprenorphine, oxycodone, morphine, fentanyl, and loperamide) in hMOR and structural similarity matrices. SD gamma scores were additionally correlated to transduction coefficients for BRET or guinea pig contractility responses respectively normalized to Met-ENK $\left(\Delta \log (\tau / \mathrm{KA})_{\mathrm{MET}}\right)$ or loperamide $(\Delta \log (\tau /$ KA) $)_{\mathrm{LOP}}$ ). Note that LOP and Met-ENK are balanced ligands that co-cluster in every data set such that differences due to normalization are simply scalar. Individual drug SD gamma scores clinically prescribed $\beta 2 A D R$ ligands used in the study (isoproterenol, norepinephrine, salbutamol, salmeterol, pindolol, carvedilol, and metoprolol) were similarly correlated to Euclidian distances separating these ligands in the $\beta 2 A D R$ similarity matrix or to transduction coefficients for BRET responses where isoproterenol was the standard.

Statistical analysis. Correlation analysis: GraphPad Prism6 was used to evaluate correlation between drug distance in cluster and the frequency of reports of adverse events.

All statistical comparisons were two-sided except when contrasting partial and whole similarity matrices.

Reporting summary. Further information on research design is available in the Nature Research Reporting Summary linked to this article.

\section{Data availability}

All data generated or analyzed in this study are included in the article and supplementary materials or provided as source data files.

\section{Code availability}

All clustering and cluster comparisons were conducted using Python 2.7.6. Complete source code is available for download at http://github.com/JonathanGallion/Benredjem-Gallion.

Received: 22 June 2018 Accepted: 6 August 2019

Published online: 09 September 2019

\section{References}

1. Lafferty-Whyte, K., Mormeneo, D. \& Del Fresno Marimon, M. Trial watch: opportunities and challenges of the 2016 target landscape. Nat. Rev. Drug Disco. 16, 10-11 (2017).

2. Lohse, M. J., Nuber, S. \& Hoffmann, C. Fluorescence/bioluminescence resonance energy transfer techniques to study G-protein-coupled receptor activation and signaling. Pharm. Rev. 64, 299-336 (2012).

3. Huang, W. et al. Structural insights into micro-opioid receptor activation. Nature 524, 315-321 (2015).

4. Kenakin, T. \& Miller, L. J. Seven transmembrane receptors as shapeshifting proteins: the impact of allosteric modulation and functional selectivity on new drug discovery. Pharm. Rev. 62, 265-304 (2010).

5. Kenakin, T. Signaling bias in drug discovery. Expert Opin. Drug Disco. 12 321-333 (2017).

6. Wacker, D., Stevens, R. C. \& Roth, B. L. How ligands illuminate GPCR molecular pharmacology. Cell 170, 414-427 (2017).

7. Luttrell, L. M., Maudsley, S. \& Gesty-Palmer, D. Translating in vitro ligand bias into in vivo efficacy. Cell Signal 41, 46-55 (2018).

8. Costa-Neto, C. M., ESLT, Parreiras \& Bouvier, M. A pluridimensional View of Biased Agonism. Mol. Pharm. 90, 587-595 (2016).

9. Pang, P. S. et al. Biased ligand of the angiotensin II type 1 receptor in patients with acute heart failure: a randomized, double-blind, placebo-controlled, phase IIB, dose ranging trial (BLAST-AHF). Eur. Heart J. 38, 2364-2373 (2017).

10. Hill, R. et al. The novel mu-opioid receptor agonist PZM21 depresses respiration and induces tolerance to antinociception. Br. J. Pharm. 175, 2653-2661 (2018).

11. Singla N. et al. A randomized, Phase IIb study investigating oliceridine (TRV130), a novel $\mu$-receptor G-protein pathway selective ( $\mu$-GPS) modulator, for the management of moderate to severe acute pain following abdominoplasty. J Pain Res. 10, 2413-2424 https://doi.org/10.2147/JPR. S137952 (2017).

12. DeWire, S. M. et al. A G protein-biased ligand at the mu-opioid receptor is potently analgesic with reduced gastrointestinal and respiratory dysfunction compared with morphine. J. Pharm. Exp. Ther. 344, 708-717 (2013).

13. Manglik, A. et al. Structure-based discovery of opioid analgesics with reduced side effects. Nature 537, 185-190 (2016).

14. Schmid, C. L. et al. Bias factor and therapeutic window correlate to predict safer opioid analgesics. Cell 171, 1165-1175 e1113 (2017).

15. Onaran, H. O. et al. Systematic errors in detecting biased agonism: analysis of current methods and development of a new model-free approach. Sci. Rep. 7, 44247 (2017).

16. Benredjem, B., Dallaire, P. \& Pineyro, G. Analyzing biased responses of GPCR ligands. Curr. Opin. Pharm. 32, 71-76 (2017).

17. Kenakin, T., Watson, C., Muniz-Medina, V., Christopoulos, A. \& Novick, S. A simple method for quantifying functional selectivity and agonist bias. ACS Chem. Neurosci. 3, 193-203 (2012).

18. Rajagopal, S. et al. Quantifying ligand bias at seven-transmembrane receptors. Mol. Pharm. 80, 367-377 (2011).

19. Lane, J. R., May, L. T., Parton, R. G., Sexton, P. M. \& Christopoulos, A. A kinetic view of GPCR allostery and biased agonism. Nat. Chem. Biol. 13, 929-937 (2017).

20. Kenakin, T. Quantifying biological activity in chemical terms: a pharmacology primer to describe drug effect. ACS Chem. Biol. 4, 249-260 (2009).

21. Raehal, K. M., Walker, J. K. \& Bohn, L. M. Morphine side effects in betaarrestin 2 knockout mice. J. Pharm. Exp. Ther. 314, 1195-1201 (2005).

22. Lee, D. D. \& Seung, H. S. Learning the parts of objects by non-negative matrix factorization. Nature 401, 788-791 (1999).

23. Forgy, Edward W. Cluster analysis of multivariate data: efficiency versus interpretability of classifications. Biometrics. 21, 768-769 (1965). JSTOR 2528559.

24. Kenakin, T. \& Christopoulos, A. Measurements of ligand bias and functional affinity. Nat. Rev. Drug Disco. 12, 483 (2013).

25. Kenakin, T. \& Christopoulos, A. Signalling bias in new drug discovery: detection, quantification and therapeutic impact. Nat. Rev. Drug Disco. 12, 205-216 (2013).

26. Gales, C. et al. Probing the activation-promoted structural rearrangements in preassembled receptor-G protein complexes. Nat. Struct. Mol. Biol. 13, 778-786 (2006).

27. Richard-Lalonde, M. et al. Conformational dynamics of Kir3.1/Kir3.2 channel activation via delta-opioid receptors. Mol. Pharm. 83, 416-428 (2013).

28. Tudashki, H. B., Robertson, D. N., Schiller, P. W. \& Pineyro, G. Endocytic profiles of delta-opioid receptor ligands determine the duration of rapid but not sustained cAMP responses. Mol. Pharm. 85, 148-161 (2014).

29. Salahpour, A. et al. BRET biosensors to study GPCR biology, pharmacology, and signal transduction. Front Endocrinol. (Lausanne) 3, 105 (2012).

30. Charfi, I. et al. Ligand- and cell-dependent determinants of internalization and cAMP modulation by delta opioid receptor (DOR) agonists. Cell Mol. Life Sci. 71, 1529-1546 (2014). 
31. Winpenny, D., Clark, M. \& Cawkill, D. Biased ligand quantification in drug discovery: from theory to high throughput screening to identify new biased mu opioid receptor agonists. Br. J. Pharm. 173, 1393-1403 (2016).

32. Black, J. W. \& Leff, P. Operational models of pharmacological agonism. Proc. R. Soc. Lond. B Biol. Sci. 220, 141-162 (1983).

33. Johnson, K., Guo, C., Gosink, M., Wang, V. \& Hauben, M. Multinomial modeling and an evaluation of common data-mining algorithms for identifying signals of disproportionate reporting in pharmacovigilance databases. Bioinformatics 28, 3123-3130 (2012).

34. Kalso, E., Edwards, J. E., Moore, R. A. \& McQuay, H. J. Opioids in chronic non-cancer pain: systematic review of efficacy and safety. Pain 112, 372-380 (2004).

35. Ballantyne, J. C. Opioid analgesia: perspectives on right use and utility. Pain. Physician 10, 479-491 (2007).

36. Rajagopal, S. Quantifying biased agonism: understanding the links between affinity and efficacy. Nat. Rev. Drug Disco. 12, 483 (2013).

37. Onaran, H. O., Rajagopal, S. \& Costa, T. What is biased efficacy? Defining the relationship between intrinsic efficacy and free energy coupling. Trends Pharm. Sci. 35, 639-647 (2014).

38. Villiger, J. W. Binding of buprenorphine to opiate receptors. Regul. guanyl nucleotides Met. Ions. Neuropharmacol. 23, 373-375 (1984).

39. Brown, S. M., Holtzman, M., Kim, T. \& Kharasch, E. D. Buprenorphine metabolites, buprenorphine-3-glucuronide and norbuprenorphine-3glucuronide, are biologically active. Anesthesiology 115, 1251-1260 (2011).

40. Galligan, J. J. \& Sternini, C. Insights into the role of opioid receptors in the GI tract: experimental evidence and therapeutic relevance. Handb. Exp. Pharm. 239, 363-378 (2017).

41. Cowie, A. L., Kosterlitz, H. W., Lydon, R. J. \& Waterfield, A. A. The effects of morphine-like substances and their antagonists on transmission at the neuroeffector junction of the myenteric plexus-longitudinal muscle preparation of the guinea-pig ileum. Br. J. Pharm. 38, 465P-466P (1970).

42. Kosterlitz, H. W. \& Waterfield, A. A. In vitro models in the study of structureactivity relationships of narcotic analgesics. Annu Rev. Pharm. 15, 29-47 (1975).

43. Yamanishi, Y., Pauwels, E. \& Kotera, M. Drug side-effect prediction based on the integration of chemical and biological spaces. J. Chem. Inf. Model 52, 3284-3292 (2012).

44. Willett P. Similarity-based virtual screening using 2D fingerprints. Drug Discov Today. 11, 1046-1053 (2006)

45. Els, C. et al. Adverse events associated with medium- and long-term use of opioids for chronic non-cancer pain: an overview of Cochrane Reviews. Cochrane Database Syst. Rev. 10, CD012509 (2017).

46. Cumming, J. G., Davis, A. M., Muresan, S., Haeberlein, M. \& Chen, H. Chemical predictive modelling to improve compound quality. Nat. Rev. Drug Disco. 12, 948-962 (2013).

47. van der Westhuizen, E. T., Breton, B., Christopoulos, A. \& Bouvier, M. Quantification of ligand bias for clinically relevant beta2-adrenergic receptor ligands: implications for drug taxonomy. Mol. Pharm. 85, 492-509 (2014).

48. Woo, A. Y. \& Xiao, R. P. beta-Adrenergic receptor subtype signaling in heart: from bench to bedside. Acta Pharm. Sin. 33, 335-341 (2012).

49. Luttrell, L. M. \& Miller, W. E. Arrestins as regulators of kinases and phosphatases. Prog. Mol. Biol. Transl. Sci. 118, 115-147 (2013).

50. Charkoudian, N. \& Rabbitts, J. A. Sympathetic neural mechanisms in human cardiovascular health and disease. Mayo Clin. Proc. 84, 822-830 (2009).

51. Hove-Madsen, L., Mery, P. F., Jurevicius, J., Skeberdis, A. V. \& Fischmeister, R. Regulation of myocardial calcium channels by cyclic AMP metabolism. Basic Res. Cardiol. 91 Suppl 2, 1-8 (1996).

52. Finney, P. A. et al. Albuterol-induced downregulation of Gsalpha accounts for pulmonary beta(2)-adrenoceptor desensitization in vivo. J. Clin. Invest. 106, 125-135 (2000).

53. Sebastian, S. et al. The in vivo regulation of heart rate in the murine sinoatrial node by stimulatory and inhibitory heterotrimeric G proteins. Am. J. Physiol. 305, R435-R442 (2013).

54. Kenakin, T. Agonists: The Measurement of Affinity and Efficacy in Functional Assays. A Pharmacology Primer, 4th Edition 85-117 (Elsevier, 2014).

55. Molinari, P. et al. Morphine-like Opiates Selectively Antagonize ReceptorArrestin Interactions. J. Biol. Chem. 285, 12522-12535 (2010).
56. Raffa, R. B. et al. The clinical analgesic efficacy of buprenorphine. J. Clin. Pharm. Ther. 39, 577-583 (2014).

57. Robertson, D. N. et al. Design and construction of conformational biosensors to monitor ion channel activation: a prototype FlAsH/BRET-approach to Kir3 channels. Methods 92, 19-35 (2016).

58. Boussif, $\mathrm{O}$. et al. A versatile vector for gene and oligonucleotide transfer into cells in culture and in vivo: polyethylenimine. Proc. Natl Acad. Sci. USA 92, 7297-7301 (1995).

59. Audet, N. et al. Differential association of receptor-G betagamma complexes with beta-arrestin2 determines recycling bias and potential for tolerance of delta opioid receptor agonists. J. Neurosci. 32, 4827-4840 (2012).

60. Carpenter, B. et al. Stan: a probabilistic programming language. J. Stat. Softw. 76, 1-32 (2017)

\section{Acknowledgements}

This research was supported by a research contract from Pfizer Inc. and grants from the Natural Sciences and Engineering Research Council of Canada (Grant 311997 to G.P.) and the Canadian Institutes of Health Research MOP 324876 (to G.P.), MOP 102630 (to M.B. and O.L.) and Foundation grant (FDN-148431) to MB. MB holds a Canada Research Chair in Signal Transduction and Molecular Pharmacology. Dr Lichtarge's research was supported by National Institutes of Health (NIH 2R01 GM066099; NIH 5R01 GM079656). B.B. was supported by a studentship from Fonds de Recherche en Santé du Québec. P.D. was supported by a MITACS fellowship. This study was supported by the Quebec Consortium on Adverse effects of pain medications, an initiative funded by the Quebec Pain Research Network (QPRN) of the Fonds de recherche du Québec Santé.

\section{Author contributions}

G.P., M.B., B.B., J.G., A.S., D.C., C.L., P.D., and O.L. conceived the study. B.B., J.G., G.P., M.B., P.D., and M.G. wrote the paper. B.B., J.C., J.G., K.N., M.G., C.L., S.J., V.L., C.S., D.P., B.M., Y.R., P.D., and E.V.W. performed experiments and/or data analysis.

\section{Additional information}

Supplementary Information accompanies this paper at https://doi.org/10.1038/s41467019-11875-6.

Competing interests: M.B., C.L., and G.P. have a patent on BRET biosensors licensed to Domain Therapeutics North America and Pfizer Inc. The remaining authors declare no competing interests.

Reprints and permission information is available online at http://npg.nature.com/ reprintsandpermissions/

Peer review information: Nature Communications thanks Stephen Duffull and other anonymous reviewer(s) for their contribution to the peer review of this work.

Publisher's note: Springer Nature remains neutral with regard to jurisdictional claims in published maps and institutional affiliations.

Open Access This article is licensed under a Creative Commons Attribution 4.0 International License, which permits use, sharing, adaptation, distribution and reproduction in any medium or format, as long as you give appropriate credit to the original author(s) and the source, provide a link to the Creative Commons license, and indicate if changes were made. The images or other third party material in this article are included in the article's Creative Commons license, unless indicated otherwise in a credit line to the material. If material is not included in the article's Creative Commons license and your intended use is not permitted by statutory regulation or exceeds the permitted use, you will need to obtain permission directly from the copyright holder. To view a copy of this license, visit http://creativecommons.org/ licenses/by/4.0/.

(C) The Author(s) 2019 